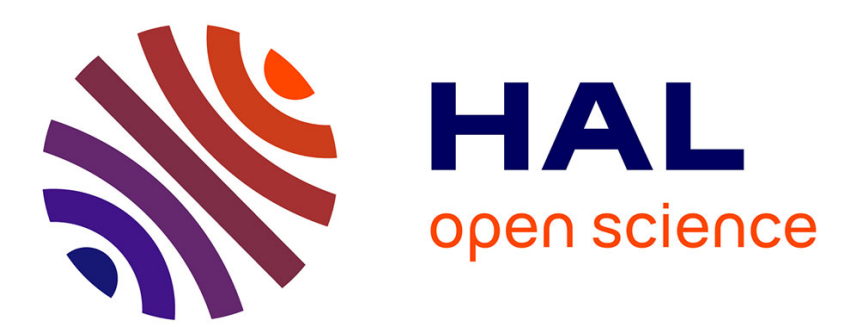

\title{
Study on the stiffness degradation and damping of pile foundations under dynamic loadings
}

\author{
Zheng Li, Sandra Escoffier, Panagiotis Kotronis
}

\section{To cite this version:}

Zheng Li, Sandra Escoffier, Panagiotis Kotronis. Study on the stiffness degradation and damping of pile foundations under dynamic loadings. Engineering Structures, 2020, 203, pp.109850. 10.1016/j.engstruct.2019.109850 . hal-02351359

\section{HAL Id: hal-02351359 \\ https://hal.science/hal-02351359}

Submitted on 15 Nov 2019

HAL is a multi-disciplinary open access archive for the deposit and dissemination of scientific research documents, whether they are published or not. The documents may come from teaching and research institutions in France or abroad, or from public or private research centers.
L'archive ouverte pluridisciplinaire HAL, est destinée au dépôt et à la diffusion de documents scientifiques de niveau recherche, publiés ou non, émanant des établissements d'enseignement et de recherche français ou étrangers, des laboratoires publics ou privés. 


\title{
Study on the stiffness degradation and damping of pile foundations under dynamic loadings
}

\author{
Zheng $\mathrm{Li}^{\mathrm{a}}{ }^{\text {,** }}$, Sandra Escoffier ${ }^{\mathrm{a}}$, Panagiotis Kotronis ${ }^{\mathrm{b}}$ \\ ${ }^{a}$ IFSTTAR, GERS Department, Geomaterials \& Models in Geotechnics Laboratory (GMG), 44341 Bouguenais, France \\ ${ }^{\mathrm{b}}$ École Centrale de Nantes, Université de Nantes, CNRS UMR 6183, GeM (Institut de Recherche en Génie Civil et Mécanique), 1 rue de la Noë, BP 92101,44321 Nantes, \\ France
}

\begin{abstract}
This paper presents a comprehensive study on the different behaviour of batter and vertical pile foundations in terms of stiffness degradation and damping properties under dynamic loadings. Firstly, dynamic centrifuge tests were carried out and the variations of translational/rotational stiffness and the associated damping properties were identified from a series of hysteretic loops. Results show that for the rocking behaviour, the presence of batter piles causes a small decrease of the rotational stiffness and a great increase of the rotational damping; for the horizontal translation behaviour, batter pile foundation has much higher horizontal stiffness and translational damping compared to the vertical foundation. Then, a set of stiffness degradation and damping curves for the translational and rotational behaviour of batter and vertical pile foundations are proposed. Finally, the proposed stiffness degradation and damping curves were validated numerically by using equivalent linear approach. The numerical validation shows a good agreement with the experimental results.
\end{abstract}

\section{Introduction}

Soil-Structure Interaction as a very important issue affecting the seismic responses of structures, has attracted much attentions from the engineering community. Many experimental results [1-4] of foundation response and nonlinear soil-structure interaction under strong seismic loadings have shown the effects of SSI of foundation system. Ahmadi et al. [5] pointed out that not only the flexibility, but also the energy dissipation of the foundation will greatly influence the inertial response of soil-structure interaction. For a rational and integrated displacementbased seismic design, SSI effects cannot be ignored [6,7].

For the SSI analysis, many approaches have been developed. Macroelement modeling, which simplifies the dynamic interaction between soil and foundation by integrating the nonlinearities into a single plastic computational unit, has attracted considerable attention [8-11]. At the same time, using spring-type models to simulate the dynamic response of soil-structure systems is popular in design practice because of their ease of use and clear physical meaning. Lu et al. [12] developed a simplified Nonlinear Sway-Rocking (NSR) model that is capable of simulating the load-displacement response of mat foundations subjected to seismic excitations. Anastasopoulos and Kontoroupi [13] proposed a nonlinear rotational spring without requiring iterations. Monotonic and cyclic pushover analyses were utilized to define the moment-rotation, damping-rotation and settlement-rotation relations. The proposed method is straightforward and efficient in predicting the rotation and settlement of shallow foundation on inelastic soil. Besides the above mentioned methods, for the analysis of SSI for shallow foundation, the Beam-on-Nonlinear Winkler Foundation (BNWF) model is also widely used for its of simplicity [14].

In recent years, more and more researchers carried out SSI analyses from the point of view of stiffness degradation and damping properties. Tileylioglu et al. [15] studied the transnational and rotational behavior of shallow foundation by large-scale model test. It is found that the damping has more pronounced energy dissipation for translational than rotational vibration modes, and increases of damping with frequency highlights the importance of radiation damping. Anastasopoulos et al. [16] studied the effectiveness of shallow soil improvement for the heavily-loaded system by rocking stiffness degradation of shallow foundation. Kokkali et al. [17] performed centrifuge tests to study the effect of shallow soil improvement on the reduction of rocking-induced settlement of shallow foundaion. Empirical equations were also proposed for shallow foundations by many researchers. For example, Gajan et al. [18] proposed a equation for the rotational stiffness degradation of shallow foundation. Gazetas et al. [19] proposed formulas and charts in a general dimensionless format which allow easy computation of the nonlinear effective rotational stiffness of foundations of any

\footnotetext{
* Corresponding author.

E-mail addresses: zheng.li@ifsttar.fr, lizheng619@hotmail.com (Z. Li), sandra.escoffier@ifsttar.fr (S. Escoffier), Panagiotis.Kotronis@ec-nantes.fr (P. Kotronis).
} 


\begin{tabular}{|c|c|}
\hline \multicolumn{2}{|c|}{ Nomenclature } \\
\hline \multicolumn{2}{|c|}{ List of symbols } \\
\hline$m_{\mathrm{t}}$ & Lumped mass of superstructure \\
\hline$m_{\mathrm{b}}$ & Lumped mass of foundation \\
\hline$u_{\mathrm{t}}$ & Horizontal translation of top mass \\
\hline$u_{\mathrm{b}}$ & Horizontal translation of foundation \\
\hline$\theta$ & Rotation of foundation \\
\hline$H_{\mathrm{t}}$ & Height of top mass \\
\hline$H_{\mathrm{b}}$ & Height of center of foundation \\
\hline$F$ & Horizontal force \\
\hline$M$ & Overturning moment \\
\hline
\end{tabular}

(reasonable) shape. The proposed formulas are suitable for the practice of design. Paolucci et al. [20-22] proposed equivalent damping curve that related to the rotational stiffness degradation curves. Studies in the similar direction are available in the literature [23-28,4]. Numerical studies concerning the stiffness degradation and damping of shallow foundaitons were also conducted by researchers. Taeseri et al. [29] studied numerically the the degradation of the rocking stiffness of shallow foundation. Adamidis et al. [30] and Pitilakis[31,32] implemented the stiffness degradation and damping curves. Equivalent linear substructure approximation was used to analyse the rocking oscillation of shallow foundation.

The existing studies mainly focused on shallow foundations. However, currently, studies for stiffness degradation and energy dissipation properties for deep foundations are very rare. To this end, this paper presents a comprehensive study of the stiffness degradation and damping for both batter and vertical pile foundations. The outline of this study is as following. The different behaviour in terms of stiffness degradation and damping properties of batter and vertical pile foundations are highlighted by a series of centrifuge tests. Then, based on the experimental results, stiffness degradation and damping curves are proposed for both batter and vertical pile foundations. Finally, numerical validation using equivalent linear approach with an iterative process is exploited to validated the proposed curves. The comparison of numerical and experimental results shows good agreement.

\section{Centrifuge tests}

Experimental campaign was carried out to investigate the rocking and lateral translation behavior of batter and vertical foundations. In this section, the preparation of centrifuge model is discussed while the experimental program will be introduced hereafter.

\subsection{Experimental set-up}

In the centrifuge tests, Fontainebleau sand (NE34) was prepared by air pluviation technique (with the relative density about $80 \%$ ). The material properties of Fontainebleau sand are listed in Table 1.

Fig. 2 shows the friction (or floating) piles and pile caps used in the experiments. The distances of piles to the boundaries of container are also indicated. Equivalent Shear Beam (ESB) container which has the inner dimensions $800 \times 340 \times 416 \mathrm{~mm}$ was used in the tests. Vertical shear rods were attached to the bottom of the container to provide complimentary shear stresses $[33,34]$. The model piles were made of

Table 1

Fontainebleau sand NE34: Material properties.

\begin{tabular}{ccccc}
\hline Soil & $e_{\min }$ & $e_{\max }$ & $e$ & $D_{\mathrm{r}}(\%)$ \\
\hline Fontainebleau NE34 & 0.51 & 0.866 & 0.58 & $80 \%$ \\
\hline
\end{tabular}

Note: $e$ :void ratio; $D_{r}$ : relative density. $u_{\mathrm{s}} \quad$ Horizontal translation of top mass due to the deformation of the column

$H \quad$ Length of the column

$\widetilde{k_{\mathrm{h}}} \quad$ Pseudo horizontal translational stiffness of foundation

$\widetilde{k_{\mathrm{r}}} \quad$ Pseudo rotational stiffness of foundation

$\widetilde{c_{\mathrm{h}}} \quad$ Pseudo horizontal translational damping coefficient of foundation

$\widetilde{c_{\mathrm{r}}} \quad$ Pseudo rotational damping coefficient of foundation

$\widetilde{D_{\mathrm{h}}} \quad$ Pseudo horizontal translational damping ratio of foundation.

$\widetilde{D_{\mathrm{r}}} \quad$ Pseudo rotational damping ratio of foundation

Table 2

Characteristics of the piles and of the pile cap, refers to Li et al. $[34,33]$.

\begin{tabular}{lll}
\hline Item & Model scale $40 \mathrm{~g}$ & Prototype scale $1 \mathrm{~g}$ \\
\hline Pile-embedded depth & $326.5 \mathrm{~mm}$ & $13.06 \mathrm{~m}$ \\
Pile-external diameter & $18 \mathrm{~mm}$ & $0.72 \mathrm{~m}$ \\
Pile-internal diameter & $15 \mathrm{~mm}$ & $0.6 \mathrm{~m}$ \\
Pile-bending stiffness & $197 \mathrm{~N} \cdot \mathrm{m}^{2}$ & $505 \mathrm{MN} \cdot \mathrm{m}^{2}$ \\
Pile-yield strength & $245 \mathrm{MPa}$ & $4245 \mathrm{MPa}$ \\
Pile cap-dimensions & $140 \mathrm{~mm} \times 40 \mathrm{~mm} \times 40 \mathrm{~mm}$ & $5.6 \mathrm{~m} \times 1.6 \mathrm{~m} \times 1.6 \mathrm{~m}$ \\
Cap-weight & $5.5 \mathrm{~N}$ & $331.24 \mathrm{kN}$ \\
\hline
\end{tabular}

aluminum alloy and had a hollow section. The distance between the pile cap and the ground surface is $28 \mathrm{~mm}$ at model scale $(1.12 \mathrm{~m}$ at prototype scale). This design was to avoid the additional soil-cap interaction $[34,33]$. The spacing of the piles was 4 times the diameter of the piles (4D) for both configurations. Table 2 gives the characteristics of the piles and the pile cap in model and prototype scales.

The following pile installation method was adopted in tests. A temporary sustaining system was used to maintain the pile group during pluviation, composed of an aluminum plate, 2 two steel rods and thin plastic wires. For both batter and vertical piles the same sustaining system was used, an example for positioning batter piles is shown in Fig. 1. The paper plate was temporarily used to verify the inclination of the piles. The thin aluminium plate (see in Fig. 1) was used to maintain precisely the inclination angle of $15^{\circ}$. The two piles were suspended by two thin steel rods in the container that are temporarily attached. Due to the falling of sand, the exact positions of piles were influenced. In order to avoid this, two thin plastic wires were attached on the tips of the two piles to provide additional supports. The sand was filled by air pluviation from the bottom up to the level of the ground surface. When the sand surface was about $5 \mathrm{~cm}$ higher than the tips of the piles, the wires were cut to free the pile tips. After the sand surface arrived at the prescribed level, the pluviation stopped and the steel rods were removed. Then, the pile cap was installed on to the pile head by tightening the bolts with a dynamic torque screw driver. Finally, the superstructure was installed on to the top of the pile cap. It should be pointed out that the adopted procedure of pluviation could minimize the "shadowing effect" ${ }^{1}$ in the zone between the two piles. However, the disturb of the homogeneity and density of sand underneath the batter piles was inevitable. For the details of installation of foundation models refers to Li et al. [33,34].

Three types of single degree of freedom superstructures were used in the tests, i.e. short, medium-tall and tall superstructures, Fig. 3(a). These superstructures were designed to have the same fixed base

\footnotetext{
${ }^{1}$ shadowing effect: during sand pluviation the presence of certain objects influence the falling of sand into the container and thus disturbs the homogeneity of the sand deposit.
} 


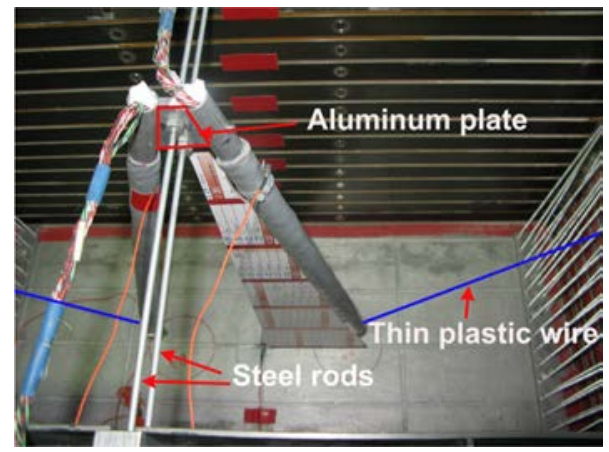

Fig. 1. Positioning the batter piles in the ESB container.

frequency; the same top mass weight; and the same total weight of the whole foundation-superstructure system. With the increase of height of the top mass, the inertial loading generated by these three superstructures varies from a horizontal force dominated to a overturning moment dominated loading case. In this study, the soil column response frequency was estimated between 3.5 and $3.7 \mathrm{~Hz}$. In order to highlight the influence of the superstructure and to distinguish it from the influence of the soil column, the frequency of the superstructure was designed to be far away from that of the soil column [33,34]. By considering the working frequency range of the shaker for sine input, the fixed base frequency of superstructure was designed to be $2.0 \mathrm{~Hz}$. The pile foundation with a tall superstructure after installation is shown in Fig. 3(b). Since the column supporting the building cannot be regarded as weightless, according to Harris and Piersol [35], approximately $23 \%$ of the weight of the column should be included in the mass at the top while the rest $77 \%$ contributes to the weight of the base. Then foundation superstructure system can be idealized as a lumped mass and column system, see Fig. 3(c). The main properties of the idealized foundation-superstructure system are summarized in Table 3.
Table 3

Main characteristics of the pile foundations with three types of superstructures.

\begin{tabular}{lccccc}
\hline & $\begin{array}{c}\text { Top } \\
\text { mass } m_{\mathrm{t}}\end{array}$ & $\begin{array}{c}\text { Found. } \\
\text { mass } m_{\mathrm{b}}\end{array}$ & $\begin{array}{c}\text { C.G. of Top } \\
\text { mass } H_{\mathrm{t}}\end{array}$ & $\begin{array}{c}\text { C.G. of } \\
\text { found. } H_{\mathrm{b}}\end{array}$ & $\begin{array}{c}\text { Column } \\
\text { stiffness }\end{array}$ \\
\hline Short & 51.2 & 64.0 tonne & $5.12 \mathrm{~m}$ & $1.92 \mathrm{~m}$ & $8090 \mathrm{kN} / \mathrm{m}$ \\
Medium-tall & tonne & & $\begin{array}{c}8.48 \mathrm{~m} \\
14.16 \mathrm{~m}\end{array}$ & & \\
Tall & & & & & \\
\hline
\end{tabular}

Note: 1 . All values are presented in prototype scale; 2 . C.G.: center of gravity.

\subsection{Experimental program}

The centrifuge tests in this study were in the framework of a project - experimental study of the performance of batter piles under dynamic

Table 4

Applied sequences of the real earthquake signals.

\begin{tabular}{lllccc}
\hline NO. & Events & Excitations & Attenuation (dB) & $\begin{array}{c}\text { PGA } \\
\left(\mathrm{m} / \mathrm{s}^{2}\right)\end{array}$ & $\begin{array}{c}\text { Arias } \\
\text { Intensity (m/ } \\
\mathrm{s})\end{array}$ \\
\hline Test 1 & $1,2,3$ & Martinique Jara & -1 & 0.9 & 0.2 \\
Test 2 & 4, 5, 6 & Northridge & -20 & 1.35 & 0.2 \\
Test 3 & $7,8,9$ & Northridge & -9 & 4.79 & 2.43 \\
Test 4 & $10,11,12$ & Kobe & -4 & 3.83 & 3.51 \\
Test 5 & $13,14,15$ & Martinique Jara & -1 & 0.9 & 0.2 \\
Test 6 & $16,17,18$ & Northridge & -20 & 1.35 & 0.2 \\
\hline
\end{tabular}

Note: 1. Earthquake records are: Martinique earthquake (Jara station EW); Northridge earthquake (Tarzana station 090) and Kobe earthquake (DAI8-G, $\mathrm{N} 43 \mathrm{~W})$ records.

2. Values of PGA (peak ground acceleration) and Arias Intensity are in prototype.

3. In order to adapt the capacity of centrifuge shaker [36], the earthquake records were attenuated. The attenuation refers to the reduction of the signal strength and is represented in decibels $(\mathrm{dB})$ - calculated as 20 times the logarithm (base 10) of the output divided by the input.
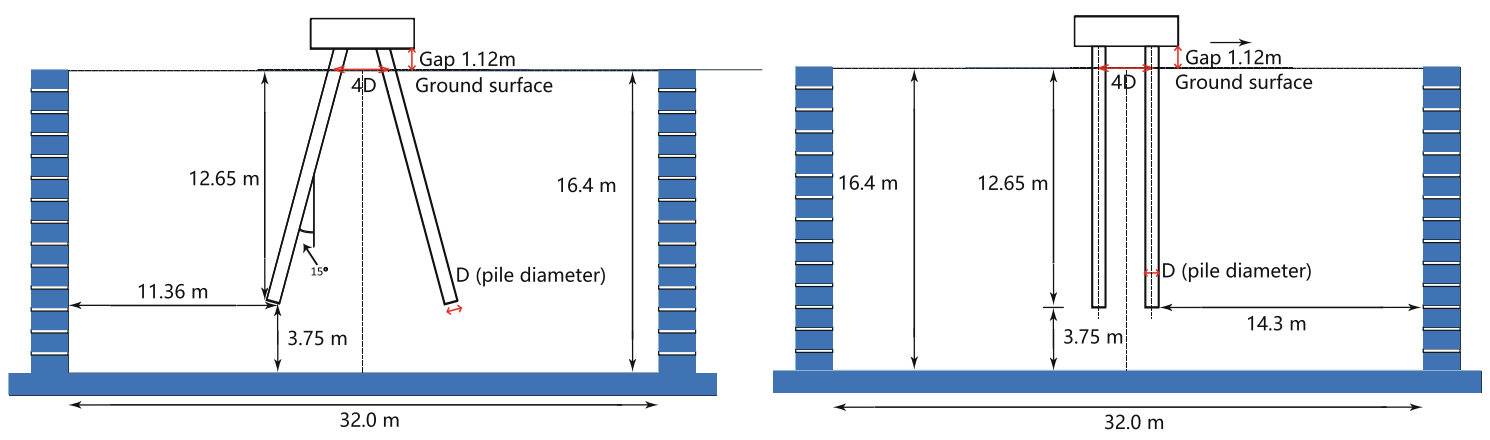

Fig. 2. Dimensions of pile foundations and ESB container (in prototype scale), refers to Li et al. [33,34].

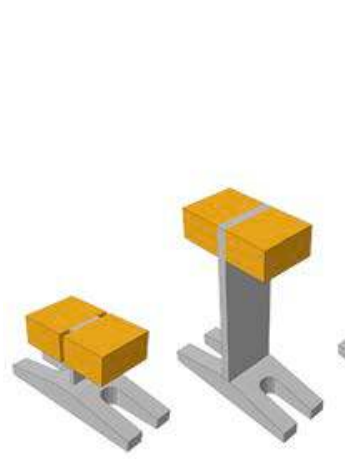

(a)

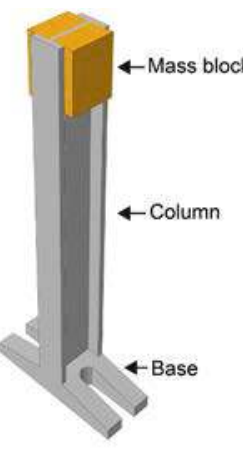

(a)

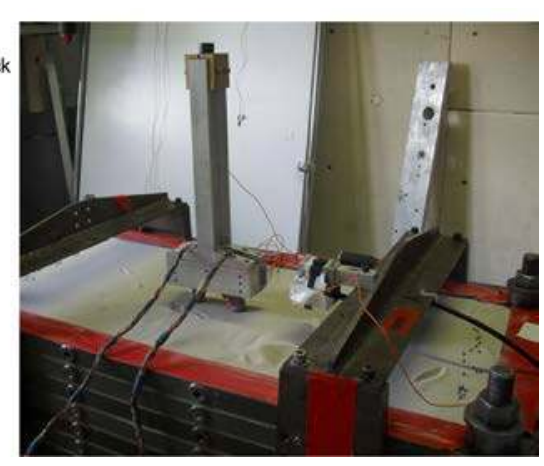

(b)

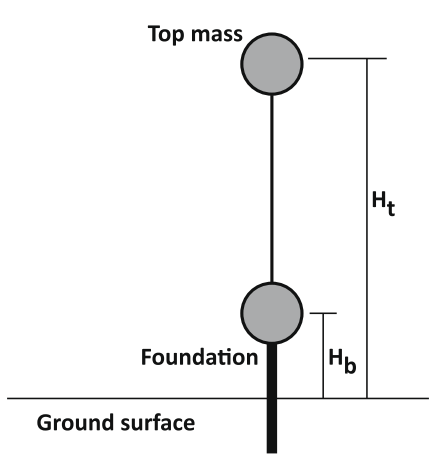

(c)

Fig. 3. (a) Superstructures; (b) Centrifuge model; (c) Idealized lumped mass model, refers to Li et al. [33,34]. 
Table 5

Applied sequence of sinusoidal signals, $2.0 \mathrm{~Hz}$ (prototype scale).

\begin{tabular}{ccccc}
\hline NO. & Events & Frequency (Hz) & PGA (g) & Arias Intensity (m/s) \\
\hline Test 1 & 1,2 & 2.0 & 0.05 & 0.34 \\
Test 2 & 3,4 & 2.0 & 0.1 & 1.35 \\
Test 3 & 5,6 & 2.0 & 0.2 & 5.37 \\
Test 4 & 7,8 & 2.0 & 0.4 & 21.38 \\
\hline
\end{tabular}

Table 6

Applied sequence of sinusoidal signals, $3.5 \mathrm{~Hz}$ (prototype scale).

\begin{tabular}{ccccc}
\hline NO. & Excitations & Frequency (Hz) & PGA (g) & Arias Intensity (m/s) \\
\hline Test 1 & 1,2 & 3.5 & 0.05 & 0.35 \\
Test 2 & 3,4 & 3.5 & 0.1 & 1.39 \\
Test 3 & 5,6 & 3.5 & 0.2 & 5.54 \\
Test 4 & 7,8 & 3.5 & 0.4 & 22.06 \\
\hline
\end{tabular}

excitations. In the first phase of this project, foundations (batter and vertical) with short and medium tall superstructures were subjected to real earthquake excitations, see Table 4 [33]; In the second phase, a taller superstructure was designed to highlight the influence of different superstructures. In order to reduce the experimental parameters, both short and taller superstructures were subjected to simple sinusoidal signals $(2.0 \mathrm{~Hz}$ and $3.5 \mathrm{~Hz}$, see Tables 5 and 6) [34]. All the loading cases are summarized in Table 7.

\section{Definitions and assumptions}

This section presents several definitions and assumptions concerning the interpretation of the experimental results and the numerical modelling in the following Sections 4 and 5.

\subsection{Calculation of rotation and lateral displacement of foundation}

In the experiments, the responses of the foundation and the superstructure are recorded by sets of accelerometers ${ }^{2}$ as shown in Fig. 4. The vertical accelerations of the pile cap are recorded by the sensors $\mathrm{CH} 12$ and $\mathrm{CH} 13$, by which the rotation of the foundation can be determined. The horizontal accelerations of the foundation and the superstructure are measured by the sensors $\mathrm{CH} 10$ and $\mathrm{CH} 11$, respectively. Sensor CH09 is far from the pile foundation and it was used to capture the farfield acceleration near the soil surface. Then the displacement of foundation and far-field soil surface can be calculated by integrating two time the accelerations in time domain. Then the relative translation of the foundation with respect to the soil surface can be calculated by the difference between $\mathrm{CH} 09$ and $\mathrm{CH} 10$ which corresponding to the displacements of the foundation and the far-field soil surface.

\subsection{Overturning moment and total horizontal force}

The overturning moment $M$ and total horizontal force $F$ acting at the foundation are calculated from the inertia forces from superstructure and the foundation using Eqs. (1) and (2). The overturning moments and horizontal forces are calculated only from the measured horizontal accelerations. Due to the small rotation of the foundation, the overturning moment caused by rotational inertia which is found less than $3 \%$ of the total overturning moment and thus was neglected. For tall superstructure, the additional overturning moment caused by $P-\Delta$ effect was also ignored.

\footnotetext{
${ }^{2}$ The type of accelerometers used in the tests is Brüel \& Kjær 4517, which is a piezoelectric shear type accelerometer with a frequency range from 1 to $20 \mathrm{kHz}$.
}

Table 7

Loading cases for different configurations of foundation-superstructure systems.

\begin{tabular}{lccc} 
Configurations & $\begin{array}{c}\text { Real } \\
\text { earthquakes }\end{array}$ & Sine $2.0 \mathrm{~Hz}$ & Sine $3.5 \mathrm{~Hz}$ \\
\hline Batter \& vertical found. with short & $\checkmark$ & $\checkmark$ & $\checkmark$ \\
Batter \& vertical found. with medium-tall & $\checkmark$ & - & - \\
Batter \& vertical found. with tall & - & $\checkmark$ & $\checkmark$ \\
\hline
\end{tabular}

Note: Frequencies are in prototype.

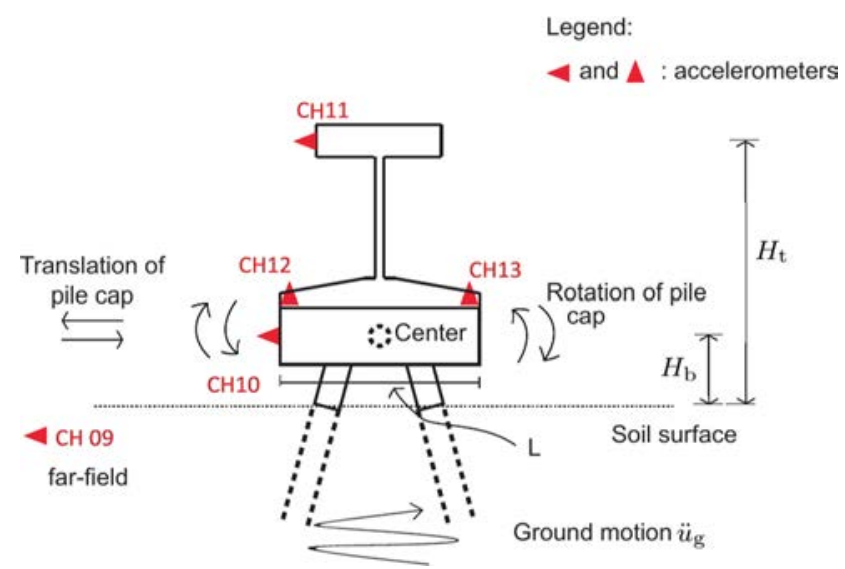

Fig. 4. Illustration of the response of the foundation and superstructure, after $\mathrm{Li}$ et al. $[33,34]$.

$M=m_{\mathrm{t}} \ddot{u}_{\mathrm{t}} H_{\mathrm{t}}+m_{\mathrm{b}} \ddot{u}_{\mathrm{b}} H_{\mathrm{b}}$

$F=m_{\mathrm{t}} \ddot{u}_{\mathrm{t}}+m_{\mathrm{b}} \ddot{u}_{\mathrm{b}}$

where $m_{\mathrm{t}}$ and $m_{\mathrm{b}}$ are the masses, $H_{\mathrm{t}}$ and $H_{\mathrm{b}}$ the C.G. and $\ddot{u}_{\mathrm{t}}$ and $\ddot{u}_{\mathrm{b}}$ the measured accelerations at the top mass and the foundation, respectively.

\subsection{Pseudo foundation stiffness and damping}

When subjected to dynamic loadings, the inertial forces acting on the foundation $f_{I}$ are balanced by the structural resisting forces $f_{S}$ and damping forces $f_{D}$. The equilibrium of forces at the C.G. of foundation can be written as Eq. (3). The matrix form is expressed by Eq. (4).

$f_{I}=f_{S}+f_{D}$

$\left[\begin{array}{c}F \\ M\end{array}\right]=\left[\begin{array}{cc}k_{\mathrm{h}} & k_{\mathrm{hr}} \\ k_{\mathrm{hr}} & k_{\mathrm{r}}\end{array}\right]\left[\begin{array}{c}u_{\mathrm{b}} \\ \theta\end{array}\right]+\left[\begin{array}{cc}c_{\mathrm{h}} & c_{\mathrm{hr}} \\ c_{\mathrm{hr}} & c_{\mathrm{r}}\end{array}\right]\left[\begin{array}{c}u_{\mathrm{b}} \\ \dot{\theta}\end{array}\right]$

where $u_{\mathrm{b}}$ and $\theta$ are the lateral displacement and rotation of foundation, respectively; $F$, the total horizontal force and $M$, the overturning moment and acting at the center of foundation, see Eqs. (2) and (1); $k_{\mathrm{h}}, k_{\mathrm{r}}$ and $k_{\mathrm{hr}}$ are the stiffness coefficients; $c_{\mathrm{h}}, c_{\mathrm{r}}$ and $c_{\mathrm{hr}}$ are the damping coefficients. Currently, in the dynamic tests and with the measured test data, it is impossible to determine directly these coefficients i.e. $k_{\mathrm{h}}, k_{\mathrm{r}}, k_{\mathrm{hr}}, c_{\mathrm{h}}, c_{\mathrm{r}}$ and $c_{\mathrm{hr}}$, especially the coupling terms $k_{\mathrm{hr}}$ and $c_{\mathrm{hr}}$. Alternatively, a uncoupled relationship could be established by assuming the coupling between foundation lateral displacement and rotation are included in some uncoupled coefficients. Based on this assumption, the coupled relationship between rotation and lateral translation of foundation in Eq. (4) can be decoupled and written as Eq. (5). Since these uncoupled terms are not the true stiffness and damping coefficients, therefore in this paper, these new coefficients are called pseudo coefficients marked with $\sim$, i.e. pseudo lateral stiffness coefficients $\widetilde{k_{\mathrm{h}}}$ and $\widetilde{k_{\mathrm{r}}}$ and pseudo rotational stiffness coefficients $\widetilde{c_{\mathrm{h}}}$ and $\widetilde{c_{\mathrm{r}}}$. In this study, these uncoupled pseudo relations facilitate the modeling of pile 


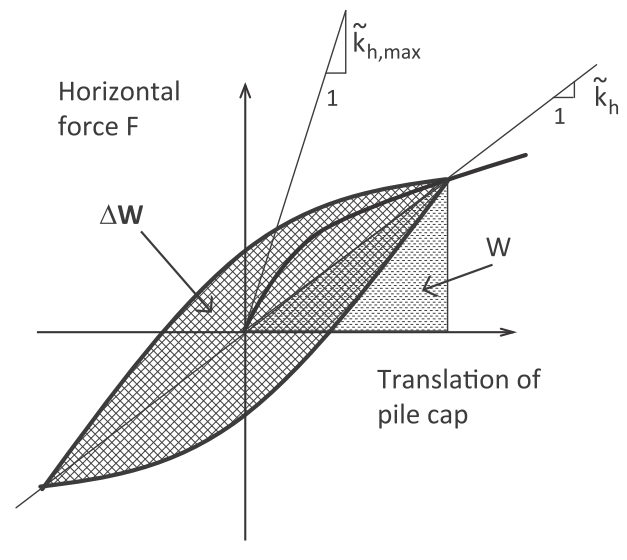

(a)

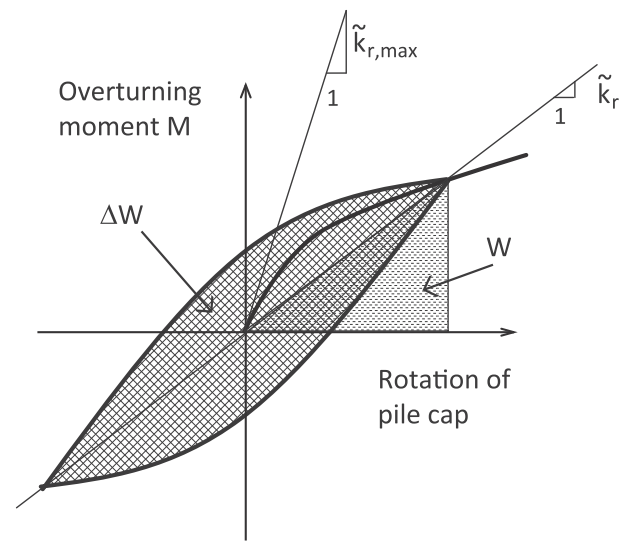

(b)

Fig. 5. (a) Equivalent lateral stiffness and damping ratio; (b) Equivalent rotational stiffness and damping ratio.

foundation by simple uncoupled nonlinear springs. However, these terms may not be applicable for the rigorous evaluation of the response of pile foundation. The determination of these coefficients will be presented hereafter.

$\left[\begin{array}{c}F \\ M\end{array}\right]=\left[\begin{array}{cc}\widetilde{k_{\mathrm{h}}} & 0 \\ 0 & \widetilde{k_{\mathrm{r}}}\end{array}\right]\left[\begin{array}{c}u_{\mathrm{b}} \\ \theta\end{array}\right]+\left[\begin{array}{cc}\widetilde{c_{\mathrm{h}}} & 0 \\ 0 & \widetilde{c_{\mathrm{r}}}\end{array}\right]\left[\begin{array}{c}\ddot{u_{\mathrm{b}}} \\ \ddot{\theta}\end{array}\right]$

For the damping of the foundation, normally, two kinds of damping are involved in the dynamic response of foundation system i.e. radiation damping and material damping. Radiation or geometric damping is a fundamental concept in modern methods of analysis of dynamic soilstructure interaction. Whereas a foundation element moves against the surrounding soil, stress waves originate at the contact sufface and spread outward. These waves carry away some of the energy tranmitted by the foundation into the soil [37-42]. Material damping is caused by inelastic behavior of the soil supporting the foundation which depends on the level of strain induced in the soil [42]. Due to the presence of the container, the stress waves were reflected back by the side walls. The energy of these waves is enclosed in the soil container which cannot be spread outward. Therefore, in this study, only material damping was considered.

\subsection{Equivalent rotational, lateral stiffness and associated damping ratio}

With the overturning moment (or horizontal force) and rotation (or translation) hysteresis loops, equivalent stiffness (rotational and transnational) and the associated damping ratio can be determined. A schematic representation is shown in Fig. 5 , where $\widetilde{k}_{\mathrm{r}, \max }$ and $\widetilde{k}_{\mathrm{r}}$ are the initial rotational and equivalent rotational stiffness, respectively; similarly, $\widetilde{k_{\mathrm{h}} \text { max }}$ and $\widetilde{k_{\mathrm{h}}}$ are the initial translational and equivalent translational stiffness, respectively.

In the dynamic tests, quiet often, the loops are in irregular shapes, shown in Fig. 6. It is difficult to determine the apexes of the enclosed loop so as to the equivalent stiffness. Alternatively, the stiffness could be obtained with Eq. (6) [43].

$\widetilde{k_{\mathrm{h}}}=\frac{\max (F)-\min (F)}{\max \left(u_{\mathrm{b}}\right)-\min \left(u_{\mathrm{b}}\right)}, \quad \widetilde{k_{\mathrm{r}}}=\frac{\max (M)-\min (M)}{\max (\theta)-\min (\theta)}$

where $u_{\mathrm{b}}$ and $F$ are the horizontal translation (or rotation) and force (or moment), respectively. The geometrical interpretation of the equivalent stiffness is shown as the line between A and B, see in Fig. 6. For an arbitrary or even irregular close loop, points A and B are often outside the loops. The damping ratio could be calculated by Eq. (7):

$D=\frac{1}{4 \pi} \frac{\Delta W}{W}$

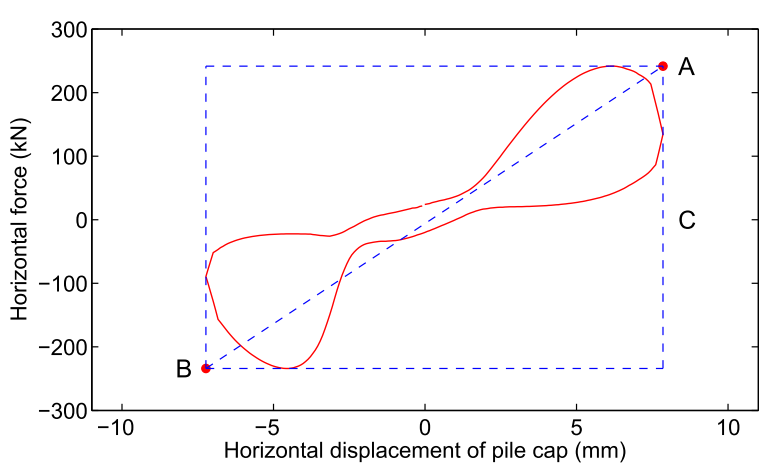

Fig. 6. A hysteretic loop extracted from experimental data.

where $\Delta W$ is the energy dissipated in a cycle and $W$ is the stored energy measured at the peak of the same cycle, respectively. Then the damping coefficients $\widetilde{c_{\mathrm{h}}}$ and $\widetilde{c_{\mathrm{r}}}$ can be calculated with the damping ratios. In addition, a method [44] to reduce the dispersion of equivalent stiffness and damping ratio from irregular loops was adopted. This method refers to collecting hysteresis loops in the same level (small range or short window) of rotation (translation) and then an average loop is found. For the whole range of rotation (translation), with the moving of this short window, less dispersed results can be obtained.

\section{Stiffness degradation and damping of pile foundations under dynamic loading}

In the dynamic centrifuge test, the soil-pile-superstructure system is loaded at the base which is close to the real loading case of ground motion. Both kinematic and inertial interaction can be included in dynamic centrifuge tests. Under dynamic loading, pile foundations move horizontally combined with rocking movements. The rocking and translational behavior of the foundations can be significantly influenced by the presence of batter piles. This section presents the different rocking and translational behavior of batter and vertical pile foundations under dynamic loadings.

\subsection{Rotational behavior of pile foundations}

First of all, the batter and vertical pile foundations under sine $2.0 \mathrm{~Hz}$ excitations are analyzed. The results are presented in terms of hysteretic loops of foundation-superstructure system under different excitation intensities, see Fig. 7. For example, Fig. 7(a) presents the results of batter pile foundation with a short superstructure under $2.0 \mathrm{~Hz}$ excitations with different peak acceleration level (from 0.05 to $0.4 \mathrm{~g}$, 


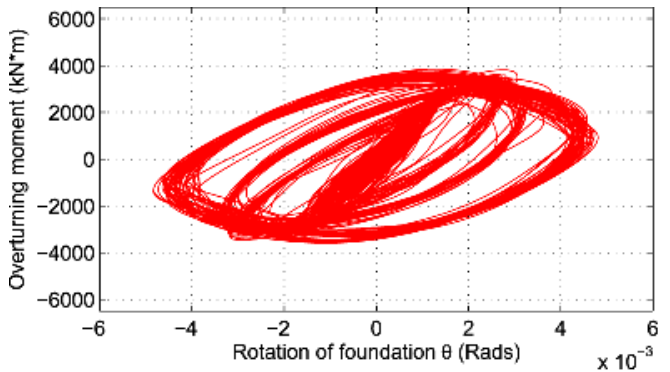

(a) IS

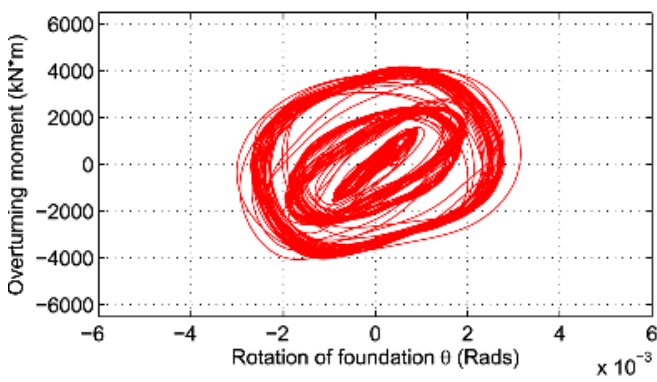

(c) ITT

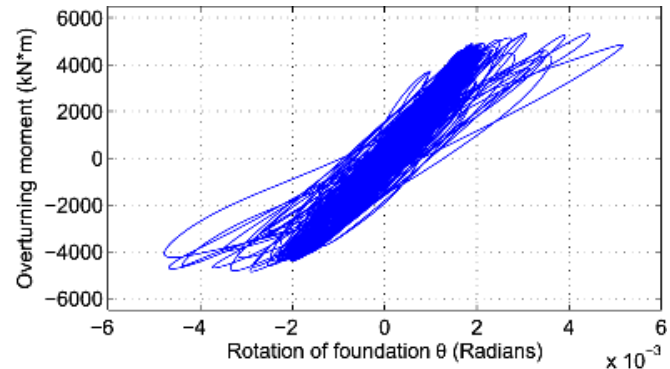

(b) VS

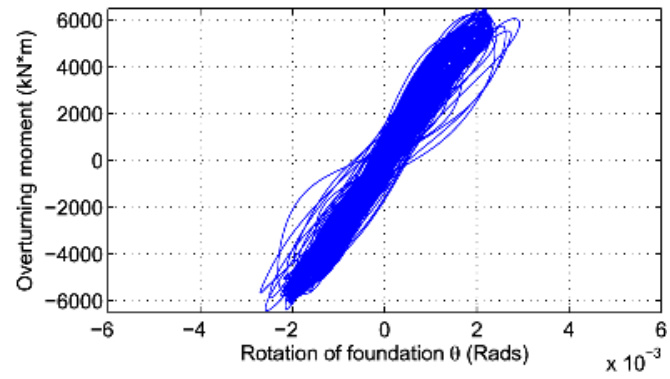

(d) VTT

Fig. 7. Rotation-moment hysteretic loops, $2.0 \mathrm{~Hz}$ sine input: (a) Batter pile foundation with short superstructure (IS, $5.12 \mathrm{~m}$ ); (b) Vertical pile foundation with short superstructure (VS, $5.12 \mathrm{~m}$ ); (c) Batter pile foundation with tall superstructure (ITT,14.16 m) and (d) Vertical pile foundation with tall superstructure (VTT, $14.16 \mathrm{~m}$ ).

Table 5). The degradation of rotational stiffness and damping for different foundation configurations are shown in Fig. 8. From the results, it is clear that the batter pile foundation has larger energy dissipation than the vertical pile group, Fig. 8 (b) and (d). However, the presence of

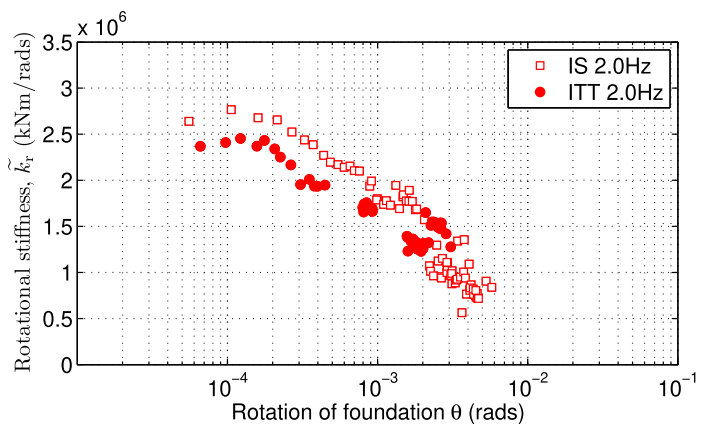

(a)

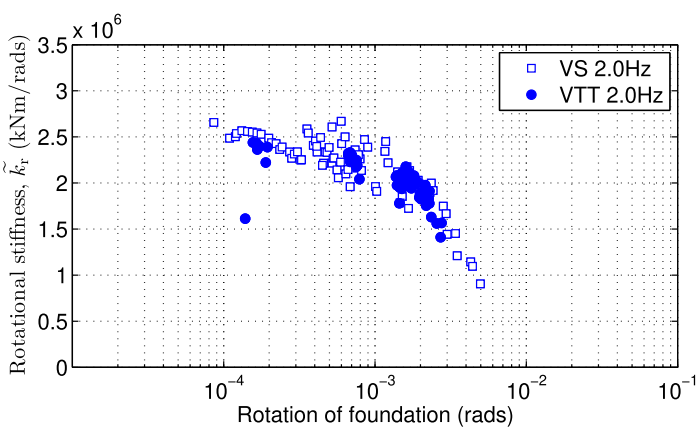

(c) batter piles does not influence so significantly the rotational stiffness degradation curve, see in Fig. 8 (a) and (c). Due to the dispersion of the data points, it is difficult to distinguish, from the current experimental data, the differences caused by the height of superstructure. The trends

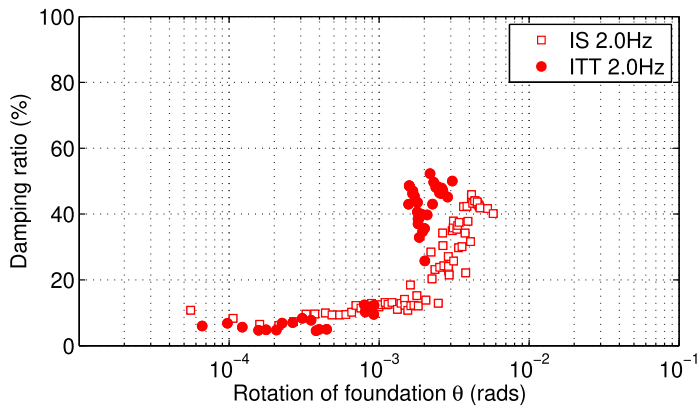

(b)

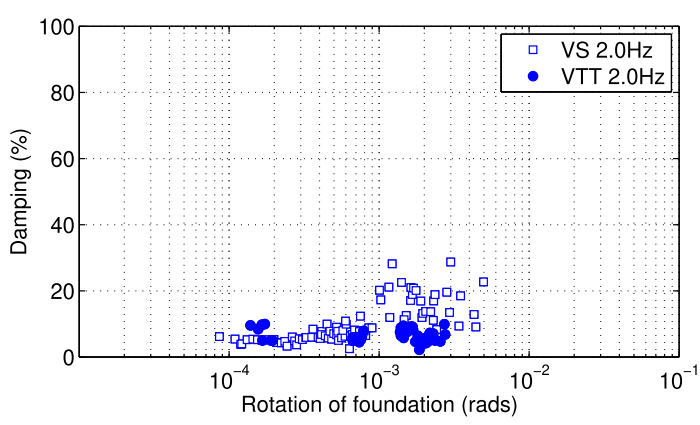

(d)

Fig. 8. Rotational stiffness degradation curve of batter and vertical pile foundations, $2.0 \mathrm{~Hz}$ input. (a) Rotational stiffness degradation curve of batter pile foundations with short (IS) and tall (ITT) superstructures and (b) Damping ratio; (c) Rotational stiffness degradation curve of vertical pile foundations with short (VS) and tall (VTT) superstructure and (d) Damping ratio. 


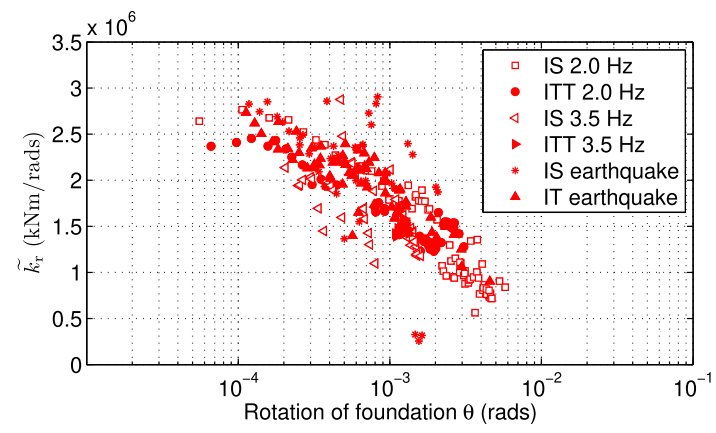

(a)

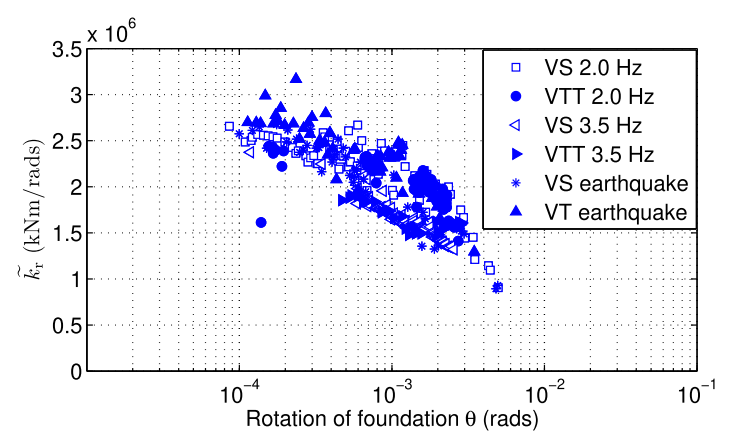

(c)

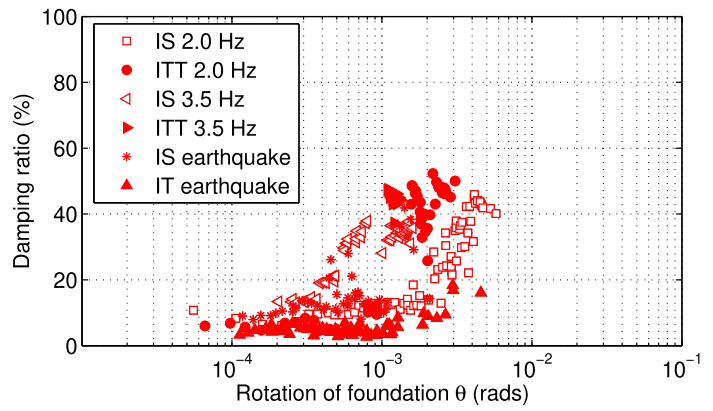

(b)

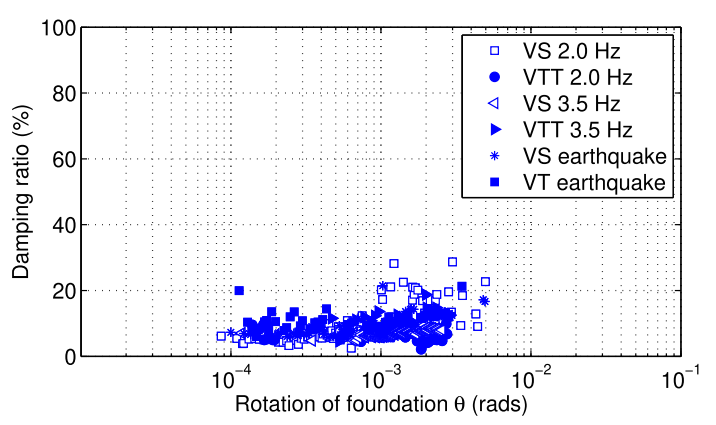

(d)

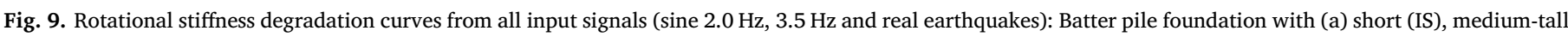

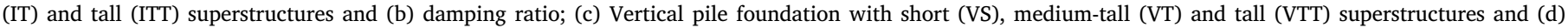
damping ratio.

for stiffness degradation and damping are almost identical for batter (or vertical) pile foundation with short and tall superstructures.

After the analyses of the results from foundation-superstructure system under sine $3.5 \mathrm{~Hz}$ and real earthquake excitations (Tables 4 and 6), it is observed that the rotational stiffness degradation and damping ratio curves follow almost the same trends in all tests, (see in Fig. 9). The results for rocking behavior of foundation-superstructure system under sine $3.5 \mathrm{~Hz}$ and real earthquake excitations are summarized in A. The good agreement between all the tests indicates that the presence of a superstructure does not influence the mechanical behavior of the foundations. The presence of batter piles has limited effect on the rotational stiffness degradation behavior, however it significantly influences the energy dissipation properties of the pile foundation system. An empirical equation is proposed to fit the data points of rotational stiffness of batter and vertical pile foundations in the following form:

$\widetilde{k_{\mathrm{r}}}=\frac{\widetilde{k_{\mathrm{r}, \max }}}{1+\alpha \theta^{\beta}}$

where $\widetilde{k_{\mathrm{r}}}$ is the secant rotational stiffness of the pile foundation; $\widetilde{k_{\mathrm{r}, \text { max }}}$ the maximum rotational stiffness; and $\alpha$ and $\beta$ constants. $\widetilde{k}_{\mathrm{r}, \max }$ is estimated empirically as $2.35 \times 10^{6} \mathrm{kN} \cdot \mathrm{m} / \mathrm{rads}$ for batter pile foundation and $2.50 \times 10^{6} \mathrm{kN} \cdot \mathrm{m} / \mathrm{rads}$ for the vertical, respectively. With these two values, curves calculated by Eq. (8) fit well with the experimental results. These initial values for rotational stiffness are also verified by FEM models ${ }^{3}$. From the FEM model, the values of $\widetilde{k}_{\text {r,max }}$ are found $2.33 \times 10^{6} \mathrm{kN} \cdot \mathrm{m} / \mathrm{rads}$ for batter pile foundation and $2.61 \times 10^{6} \mathrm{kN} \cdot \mathrm{m} /$ rads for the vertical, which are very close to the empirically estimated

\footnotetext{
${ }^{3}$ FEM models of pie foundations were carried out using advanced hypoplastic constitutive model $[45,46]$ and the numerical modeling technique proposed by Li et al. [11] was adopted.
}

values. For the numerical determination of the initial stiffness, the FEM model utilized advanced soil constitutive law with soil stiffness in small-strain range. It means alternatively a simpler way of estimation of these values by linearly elastic model could be adopted.

For both batter and vertical configurations $\alpha$ and $\beta$ are $5.0 \times 10^{3}$ and 1.4 , respectively. The maximum rotational stiffness for the batter pile foundation is lower than that of the vertical pile foundation due to the presence of batter piles. The fitting curves together with all the data points are plotted in Fig. 10.

As for the damping properties, the damping ratio curves for batter and vertical foundation are linked with their rotational stiffness degradation curves. The proposed form for the fitting equation is the following:

$\widetilde{D_{\mathrm{r}}}=\widetilde{D_{\mathrm{r}, \max }}\left(m\left(\frac{\widetilde{k_{\mathrm{r}}}}{\widetilde{k_{\mathrm{r}, \max }}}\right)^{1.1}-n\left(\frac{\widetilde{k_{\mathrm{r}}}}{\widetilde{k_{\mathrm{r}, \max }}}\right)+1\right)$

where $\widetilde{D_{\mathrm{r}}}$ is the rotational damping ratio; $\widetilde{D_{\mathrm{r}} \text { max }}$ is the maximum rotational damping ratio; $m$ and $n$ are fitting parameters. $\widetilde{D_{r}, \max }$ is estimated around $60 \%$ for batter pile foundation and $25 \%$ for vertical pile foundation, respectively. For both cases $m$ and $n$ are 0.63 and 1.5, respectively. Nonlinear FEM simulations were also carried out to estimate the maximum damping ratios. Results show the maximum damping ratios are about $40 \%$ and $20 \%$ for batter and vertical pile groups. Different from the good estimation of initial stiffness, the nonlinear FEM simulation cannot well predict the maximum damping ratio. To this end, in this study, the empirically estimated damping ratios are used to have the best fitting curves with the experimental data. The relationships between rotational stiffness and damping ratio for the batter and vertical foundations together with the fitting curves are plotted in Fig. 11. The main constants for Eqs. (8) and (9) are summarized in Table 8.

Finally the damping ratio curves for batter and vertical pile 


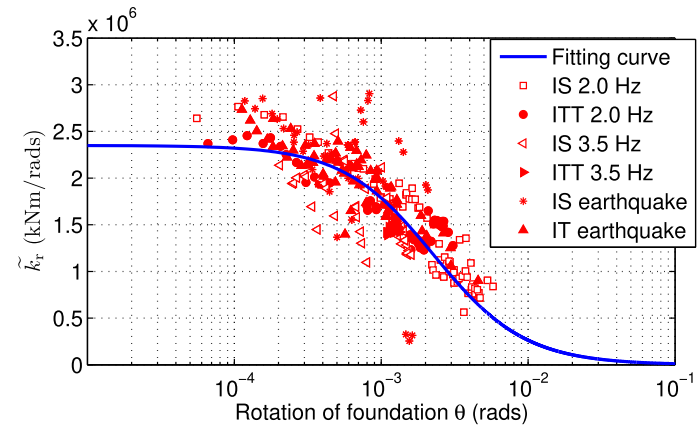

(a)

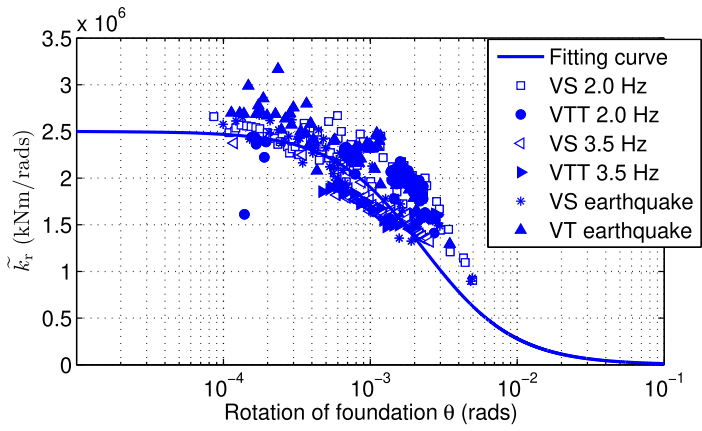

(b)

Fig. 10. Rotational stiffness degradation fitting curves: (a) Batter pile foundation and (b) Vertical pile foundation.

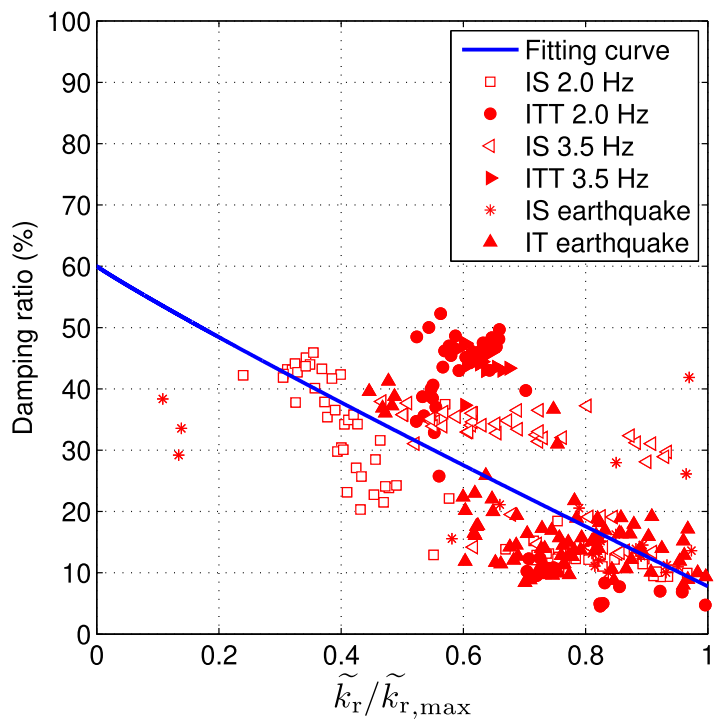

(a)

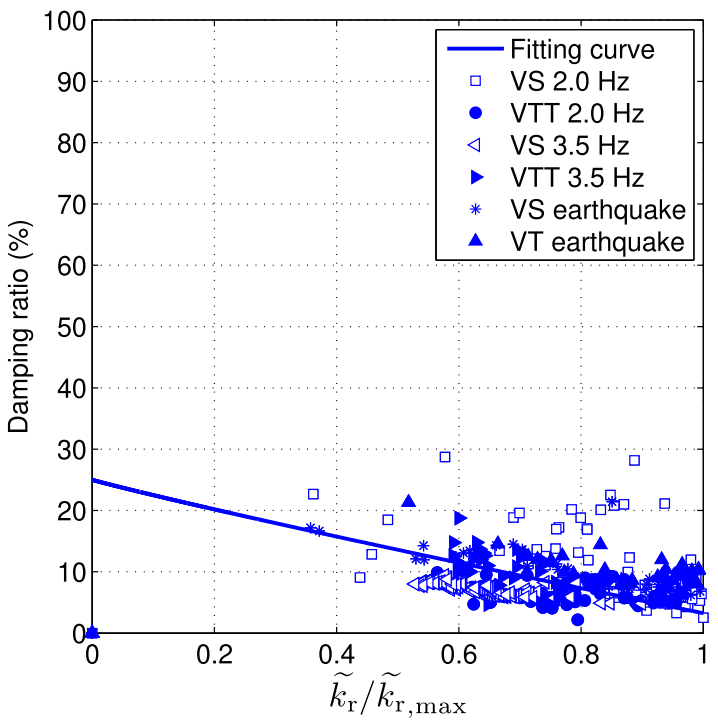

(b)

Fig. 11. Damping ratio in function of normalized rotational stiffness: (a) Batter pile foundation and (b) Vertical pile foundation.

Table 8

Main constants for rotational stiffness degradation and damping curves of batter and vertical pile foundation.

\begin{tabular}{lllllll}
\hline & $\widetilde{k}_{\mathrm{r}, \max }$ & $\alpha$ & $\beta$ & $\widetilde{D_{\mathrm{r}, \max }}$ & $m$ & $n$ \\
\hline Batter pile found. & $2.35 \times 10^{6} \mathrm{kN} \cdot \mathrm{m} / \mathrm{rads}$ & $5.0 \times 10^{3}$ & 1.4 & $60 \%$ & 0.63 & 1.5 \\
Vertical pile found. & $2.50 \times 10^{6} \mathrm{kN} \cdot \mathrm{m} / \mathrm{rads}$ & & & $25 \%$ & &
\end{tabular}

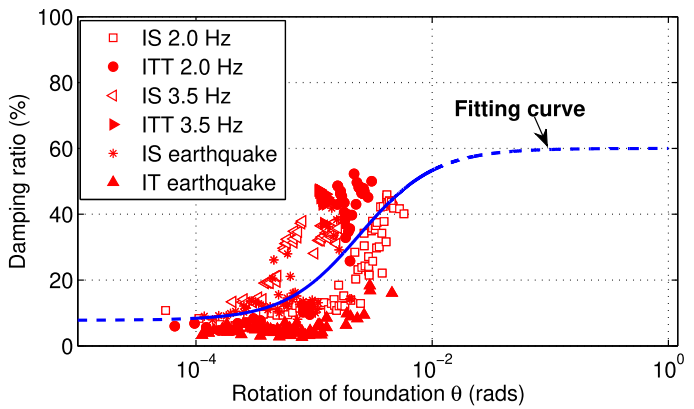

(a) foundations are obtained and plotted in Fig. 12. It should be acknowledged that the values of $\widetilde{D_{r}, \max }$ and parameters $m$ and $n$ are empirically determined to ensure that Eq. (9) fits well with the experimental data in its range. The accuracy cannot be guaranteed if the fitting curves are out of the range of the data points i.e. rotation $<=10^{-4}$ and $>=10^{-2}$, where the curves are marked by dashed lines in Fig. 12 .

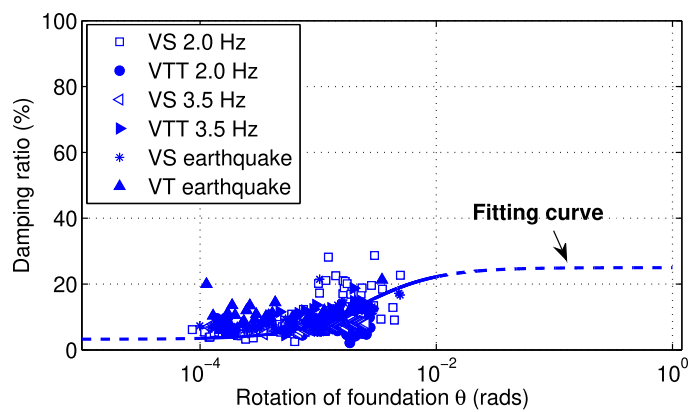

(b)

Fig. 12. Damping ratio in function of rotation of foundation: (a) Batter pile foundation and (b) Vertical pile foundation. 


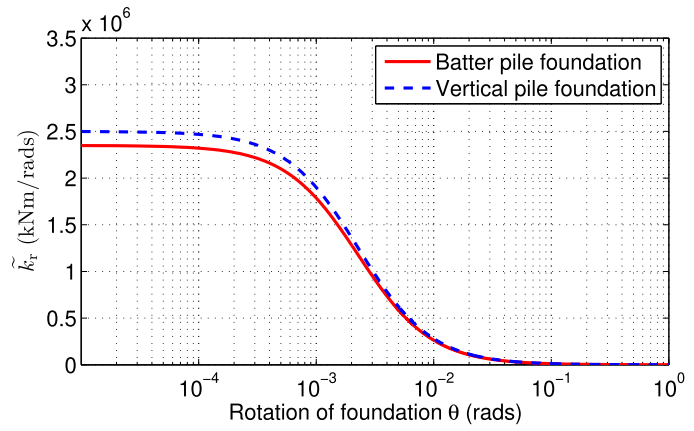

(a)

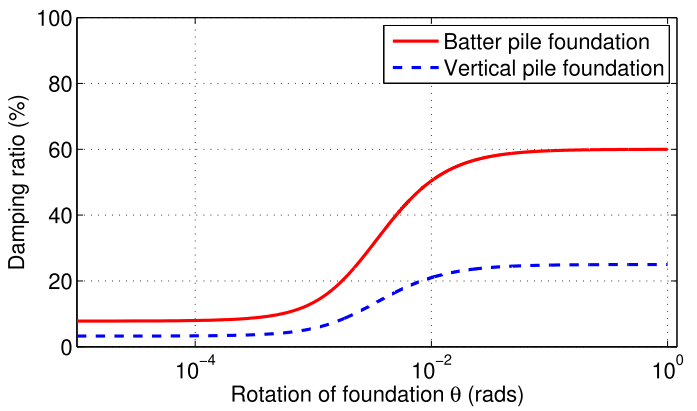

(b)

Fig. 13. Comparison of rocking behaviour of batter and vertical foundations: (a) degradation of rotational stiffness and (b) damping ratio.

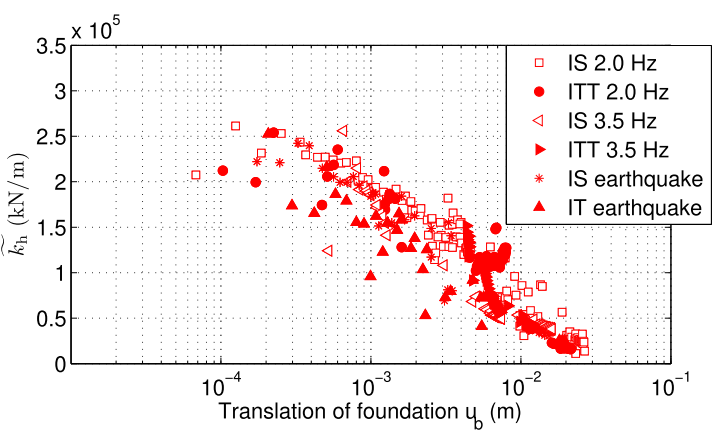

(a)

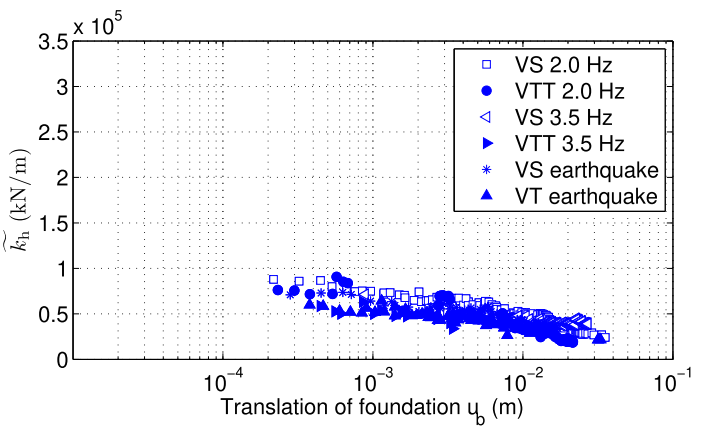

(c)

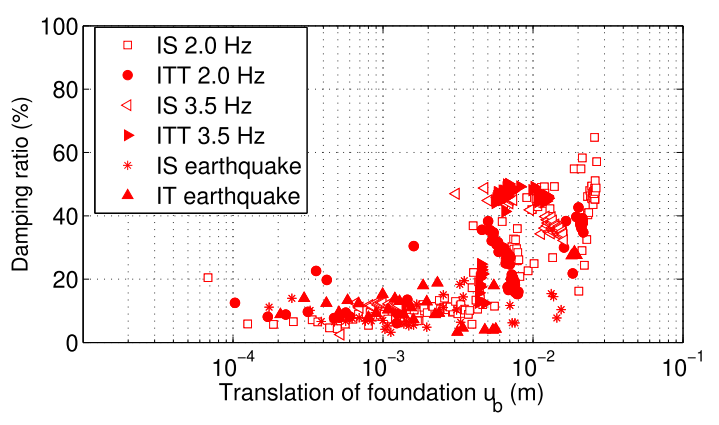

(b)

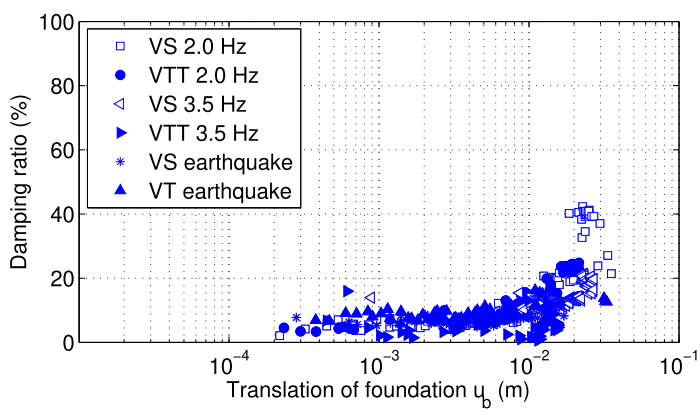

(d)

Fig. 14. Translational stiffness degradation curves for all input signals (sine $2.0 \mathrm{~Hz}, 3.5 \mathrm{~Hz}$ and real earthquakes): (a) Batter pile foundation with short and tall superstructures and (b) damping ratio; (c) Vertical pile foundation with short and tall superstructure and (d) damping ratio.

The comparison of stiffness degradation and damping between batter and vertical pile foundations is summarized in Fig. 13. It can be seen that the rotational stiffness is not significantly influenced by the presence of the batter piles. However, batter pile foundation has larger energy dissipation capacity.

\subsection{Lateral behaviour of pile foundations}

Similar as the rotational behavior of foundation-superstructure system, the horizontal behavior of foundation is analyzed using hysteretic loops (results for horizontal translation and force hysteretic loops are summarized in Appendix B).

Plotting the results from all different dynamic loadings together, see in Fig. 14, it is obvious that they have very similar tendencies. This confirms the former conclusion that the behavior of foundations is not influenced by the superstructures. Following the same procedure in the previous sections, the horizontal translation stiffness degradation are fitted by empirical equations:

$\widetilde{k_{\mathrm{h}}}=\frac{\widetilde{k_{\mathrm{h}, \max }}}{1+\alpha u^{\beta}}$

where $\widetilde{k_{\mathrm{h}}}$ is pseudo horizontal translational stiffness; $\widetilde{k_{\mathrm{h}, \max }}$ is the maximum pseudo horizontal translation stiffness; $u$ is the horizontal translation; $\alpha$ and $\beta$ are fitting parameters. The maximum pseudo horizontal stiffnesses are estimated as approximately $2.3 \times 10^{5} \mathrm{kN} / \mathrm{m}$ and $0.75 \times 10^{5} \mathrm{kN} / \mathrm{m}$ for batter and vertical pile foundation, respectively. These initial values are also verified by FEM models. From the FEM model, the values of $\widetilde{k_{h}, \text { max }}$ are found $2.4 \times 10^{5} \mathrm{kN} / \mathrm{m}$ for batter pile foundation and $0.97 \times 10^{5} \mathrm{kN} / \mathrm{m}$ for the vertical, which agree well with the empirically estimated values. $\alpha$ and $\beta$ for both cases are 200 and 1.05 respectively. For the damping ratio, a similar equation is proposed in function of the degradation of translational stiffness: 


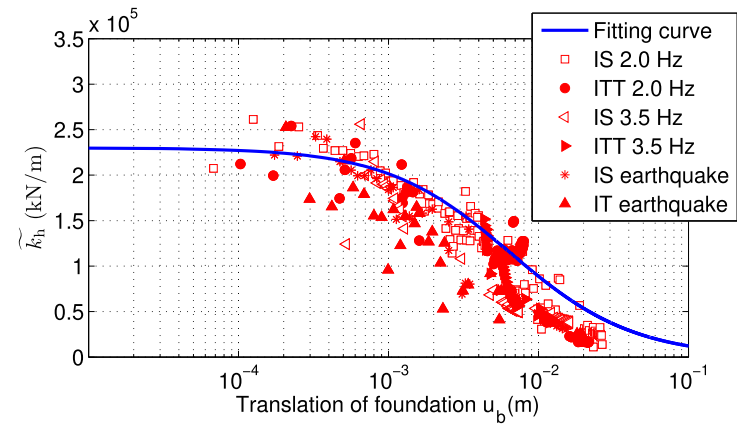

(a)

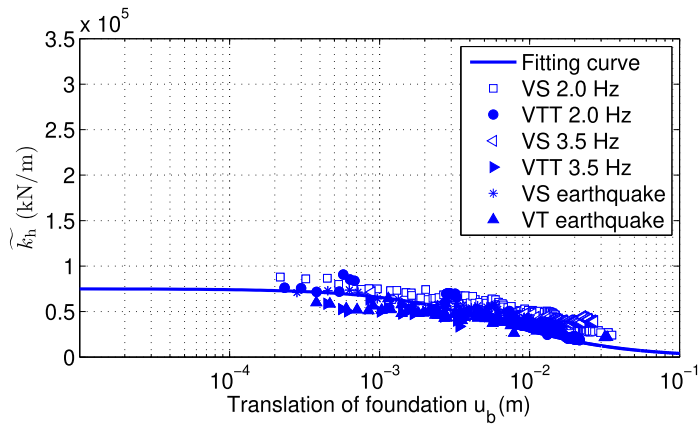

(b)

Fig. 15. Fitted horizontal translation stiffness degradation curves: (a) Batter pile foundation and (b) Vertical pile foundation.

$\widetilde{D_{\mathrm{h}}}=\widetilde{D_{\mathrm{h}, \max }}\left(m\left(\frac{\widetilde{k_{\mathrm{h}}}}{\widetilde{k_{\mathrm{h}, \text { max }}}}\right)^{2}-n\left(\frac{\widetilde{k_{\mathrm{h}}}}{\widetilde{k_{\mathrm{h}, \text { max }}}}\right)+1\right)$

where $\widetilde{D_{\mathrm{h}}}$ is the damping ratio associated with the translation of the pile foundation; $\widetilde{D_{\mathrm{h}} \text { max }}$ is the maximum damping ratio; $m$ and $n$ are two fitting parameters. $\widetilde{D_{\mathrm{h}} \text { max }}$ is estimated around $60 \%$ for batter pile foundation and $35 \%$ for vertical pile foundation. $m$ and $n$ for both cases are 0.88 and 1.8 respectively. Nonlinear FEM simulations found the maximum damping ratios were about $35 \%$ and $25 \%$ for batter and vertical pile groups. These values cannot fit well with the experimental data. In this study, the empirically estimated damping ratios are used to have the best fitting curves with the experimental data. Figs. 15-17 show the comparisons of the fitting curves with the all the data points. As it was explained before, with empirical parameters, Eq. (11) fits well within the range of experimental data. For other ranges, accuracy cannot be guaranteed and marked by dashed curves, see Fig. 17. Table 9 summarized the parameters for the empirical equation for horizontal translational behavior of foundations.

Finally, by comparison, it can be concluded from Fig. 18 that batter piles greatly increase the horizontal stiffness of foundation system by around 3.0 times; while batter pile foundation also have greater damping ratio for horizontal behavior.

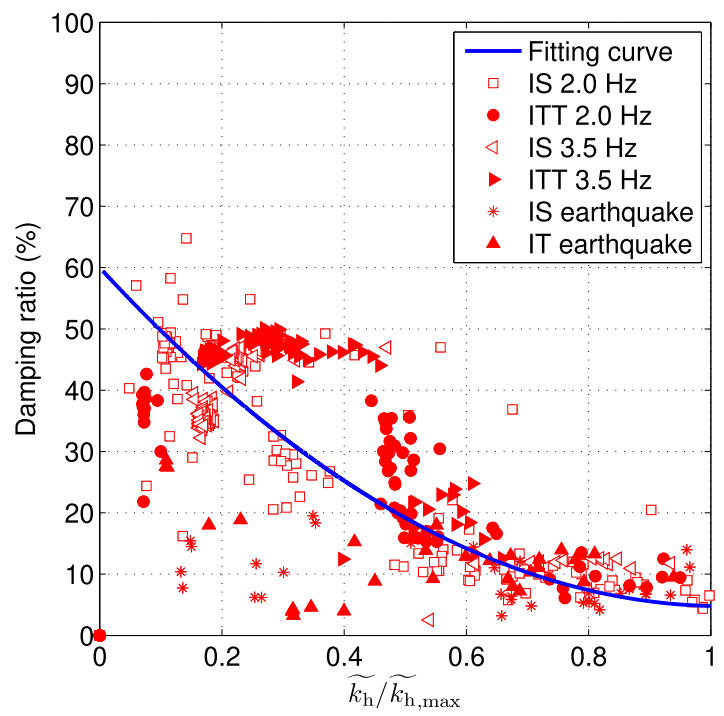

(a)

\subsection{Summary}

In this study, a comprehensive experimental study of the performance of batter and vertical pile foundations is carried out. For the rocking behavior, the presence of the batter piles increases the rotational damping ratio without losing so much rotational stiffness; for the horizontal translation behavior. Batter piles have a more important horizontal stiffness than the vertical and energy dissipation ability. Due to the inclinations, batter piles provides substantial horizontal resistance to the foundation. When subjected to horizontal loading which causes rotation and translation of foundation, batter piles may have much more complicated nonlinear soil-pile interaction in both axial and lateral directions. This is in accordance with the numerical results from 3D nonlinear FEM simulations of Li et al. [47]. It is found that under cyclic horizontal loading, batter pile has more important axial reaction force and energy loss due to plastic deformation. The current results from a relative large number of tests show that the behavior of the pile foundation system is not influenced by the type of superstructures.

\section{Modelling of nonlinear behaviour of pile foundations}

Based on the proposed stiffness degradation and damping curves, Equivalent Linear Approach (ELA) was used to validate the feasibility of using these curves to analyse the nonlinear response of pile

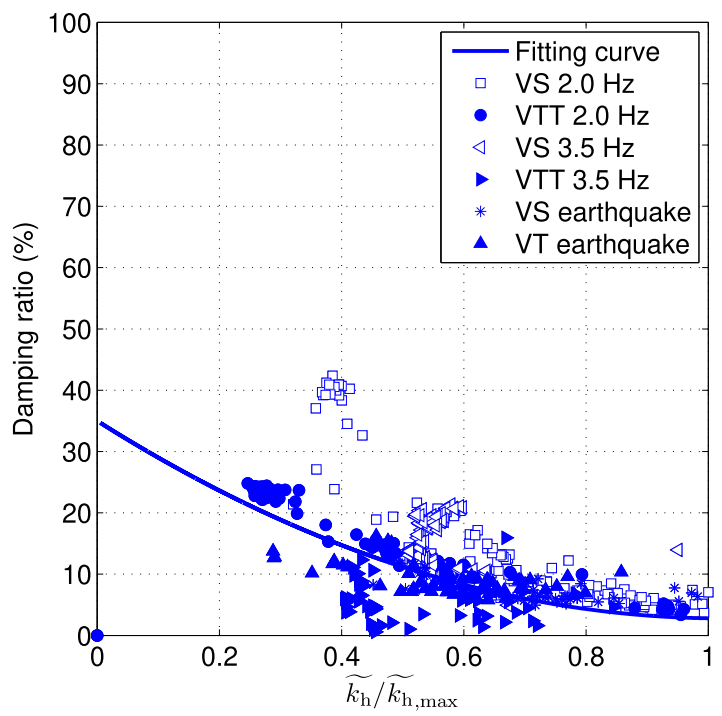

(b)

Fig. 16. Damping ratio in function of normalized translational stiffness: (a) Batter pile foundation and (b) Vertical pile foundation. 


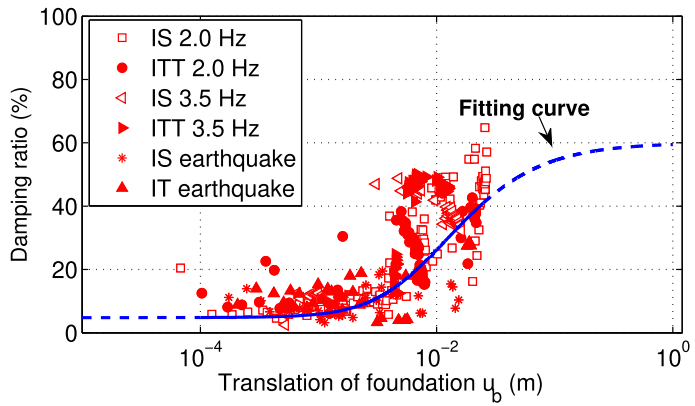

(a)

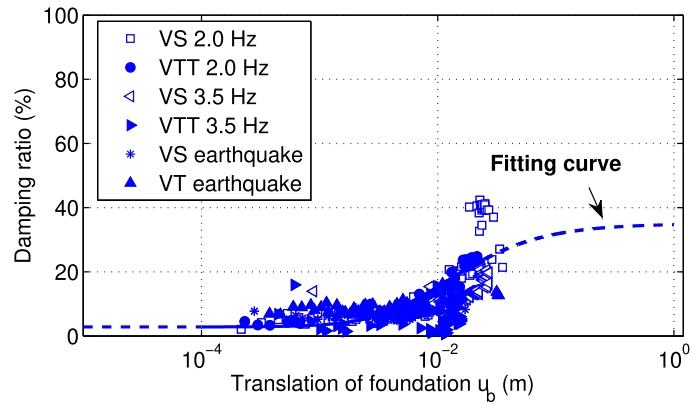

(b)

Fig. 17. Damping ratio in function of horizontal translation of foundation: (a) Batter pile foundation and (b) vertical pile foundation.

Table 9

Main constants for horizontal stiffness degradation and damping curves of batter and vertical pile foundation.

\begin{tabular}{lllllll}
\hline & $\widetilde{k_{\mathrm{h}, \max }}$ & $\alpha$ & $\beta$ & $\widetilde{D_{\mathrm{h}, \max }}$ & $m$ & $n$ \\
\hline Batter pile found. & $2.3 \times 10^{5} \mathrm{kN} / \mathrm{m}$ & 200 & 1.05 & $60 \%$ & 0.88 & 1.8 \\
Vertical pile found. & $0.75 \times 10^{5} \mathrm{kN} / \mathrm{m}$ & & & $35 \%$ & & \\
\end{tabular}

foundations. The main numerical approach and validations will be presented hereafter.

The dynamic equilibrium of the system is shown in Fig. 19. The foundation system can be idealized as springs and dampers which control the translational and rotational behaviour. The dynamic equilibrium of this simplified foundation-superstructure system can be described by the following set of equations, Eq. (12):

$M \ddot{u}+C \dot{u}+K u=p$

where

$$
\begin{aligned}
M & =\left[\begin{array}{ccc}
m_{\mathrm{t}} & 0 & 0 \\
0 & m_{\mathrm{b}} & 0 \\
0 & 0 & J_{\mathrm{t}}
\end{array}\right] \\
C & =\left[\begin{array}{ccc}
0 & 0 & 0 \\
0 & \widetilde{c_{\mathrm{h}}} & 0 \\
0 & 0 & \widetilde{c_{\mathrm{r}}}
\end{array}\right] \\
K & =\left[\begin{array}{ccc}
k & -k & -k H \\
-k & k & k H \\
-k H & k H & k H^{2}
\end{array}\right]+\left[\begin{array}{ccc}
0 & 0 & 0 \\
0 & \widetilde{k_{\mathrm{h}}} & 0 \\
0 & 0 & \widetilde{k}_{\mathrm{r}}
\end{array}\right]
\end{aligned}
$$$$
u=\left[\begin{array}{lll}
u_{\mathrm{t}} & u_{\mathrm{b}} & \phi
\end{array}\right]^{T}
$$

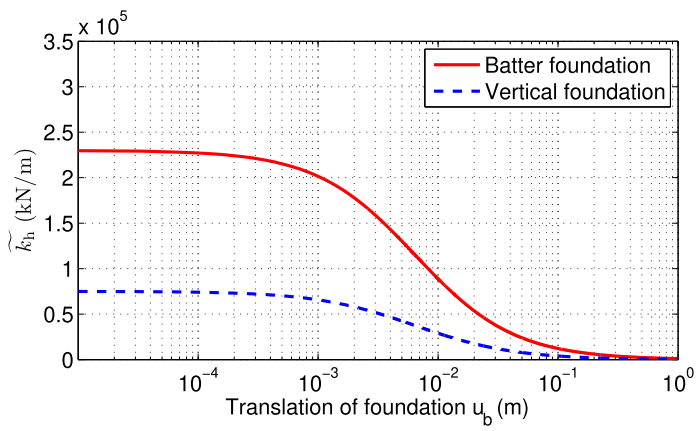

(a)

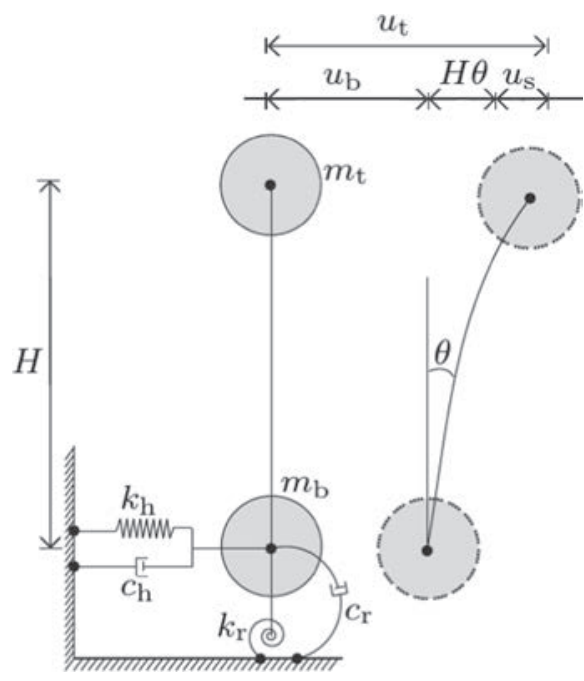

$u_{\mathrm{s}}$ is the horizontal translation of top mass due to the deformation of the column.

Fig. 19. Computational model for nonlinear dynamic soil-structure interaction analysis.

$p=\left[\begin{array}{lll}-m_{\mathrm{t}} \ddot{\mathrm{u}}_{\mathrm{g}}-m_{\mathrm{b}} \ddot{u}_{\mathrm{g}} 0 & 0\end{array}\right]^{T}$

where, $m_{\mathrm{t}}$ and $m_{\mathrm{b}}$ are the lumped masses of superstructure and foundation, respectively; $u_{\mathrm{t}}$ and $u_{\mathrm{b}}$ are the horizontal translation of top mass and foundation, respectively, relative to the ground surface; $\theta$ is the rotation of foundation; $H$, length of the column; $\widetilde{k_{h}}$, pseudo horizontal translational stiffness of foundation; $\widetilde{k_{\mathrm{r}}}$, pseudo rotational stiffness of foundation; $\widetilde{c_{h}}$, pseudo horizontal translational damping coefficient of foundation, $\widetilde{c_{\mathrm{h}}}=\widetilde{D_{\mathrm{h}}} \cdot 2 \sqrt{\widetilde{k}_{\mathrm{h}} m_{\mathrm{t}}} ; \widetilde{c_{\mathrm{r}}}$, pseudo rotational damping coefficient

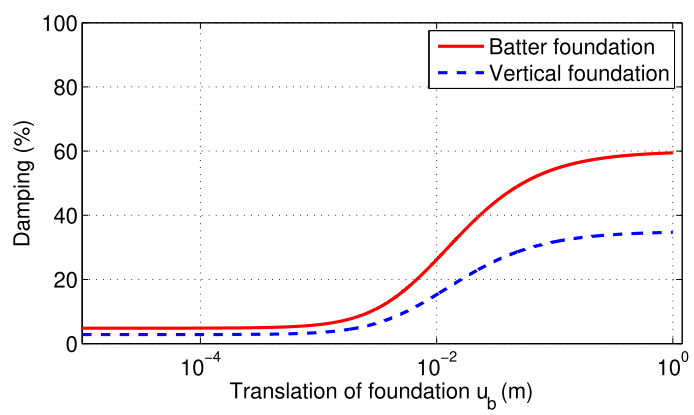

(b)

Fig. 18. Comparison of translation behavior of batter pile foundation and vertical foundation: (a) degradation of rotational stiffness and (b) damping ratio. 


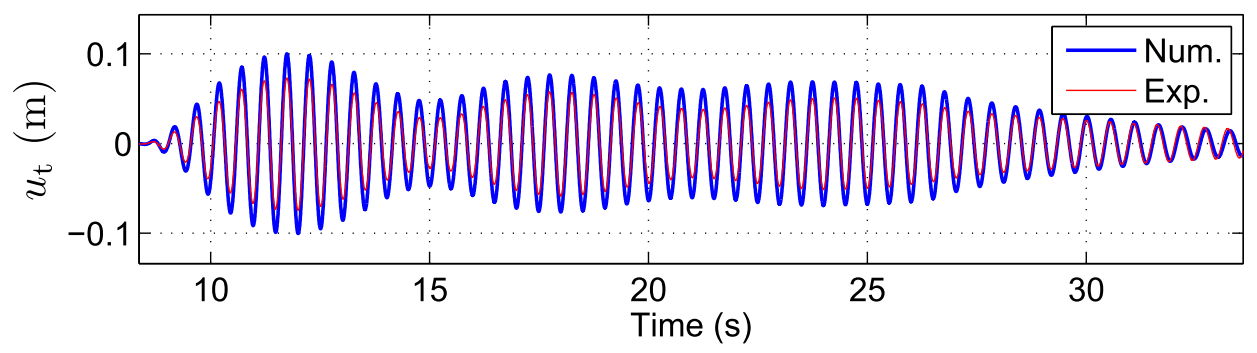

Fig. 20. Simulation of batter pile foundation with short superstructure $(5.12 \mathrm{~m}$, sine $2 \mathrm{~Hz}$ input). Comparison of displacement history of top mass.

of foundation, $\widetilde{c_{\mathrm{r}}}=\widetilde{D_{\mathrm{r}}} \cdot 2 \sqrt{\widetilde{k}_{\mathrm{r}}} ; \widetilde{D_{\mathrm{h}}}$, pseudo horizontal translational damping ratio of foundation; $\widetilde{D_{\mathrm{r}}}$, pseudo rotational damping ratio of foundation; $J_{\mathrm{t}}$, moment of inertia of foundation; $k$ is the lateral stiffness of the column. In the governing equation of the system Eq. (12), the damping in the column is ignored.
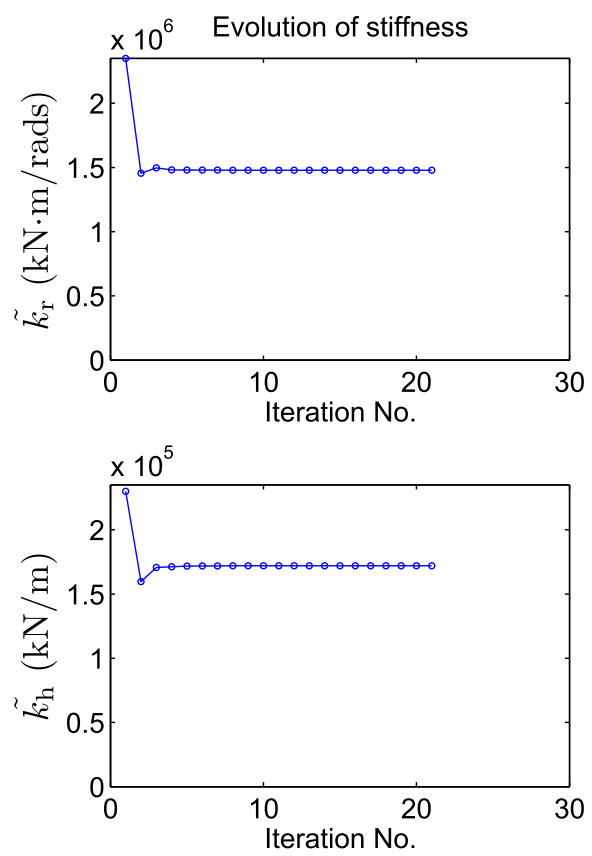

In order to implement the foundation stiffness degradation and damping curves, Equivalent Linear Approach (ELA) was adopted. This method was initially proposed by Schnabel et al. [48] for analyzing free-field responses of ground under seismic actions. In this study, in stead of using the recursion formulas in frequency domain, step-by-step integration in time domain was adopted (Newmark-Beta method
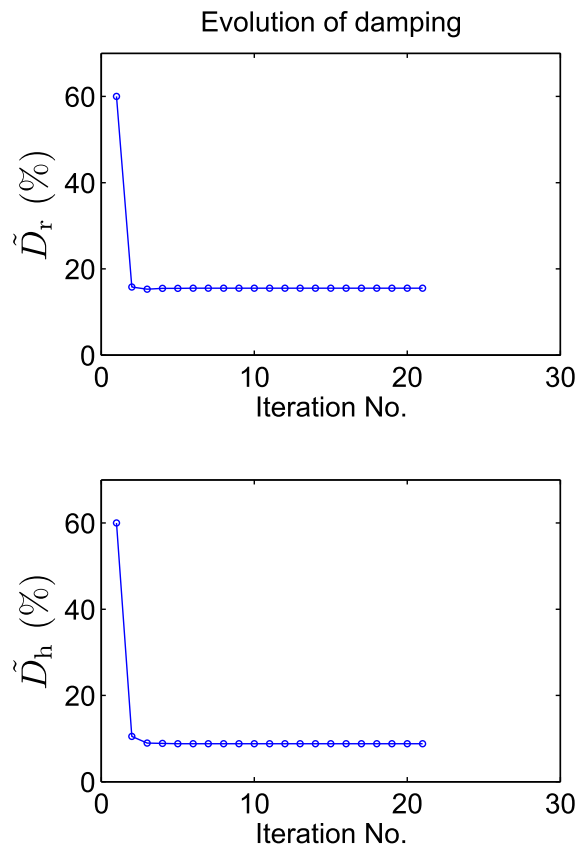

(a)

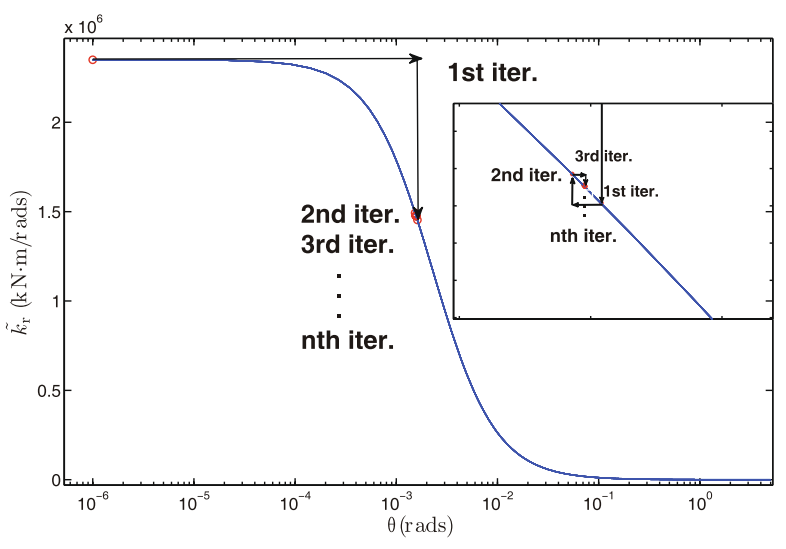

(b)

Fig. 21. Simulation of batter pile foundation with short superstructure ( $5.12 \mathrm{~m}$, sine $2 \mathrm{~Hz}$ input). (a) Evolution history of stiffness and damping ratio from their initial values; (b) An example of iterations of $\widetilde{k_{\mathrm{r}}}$. 


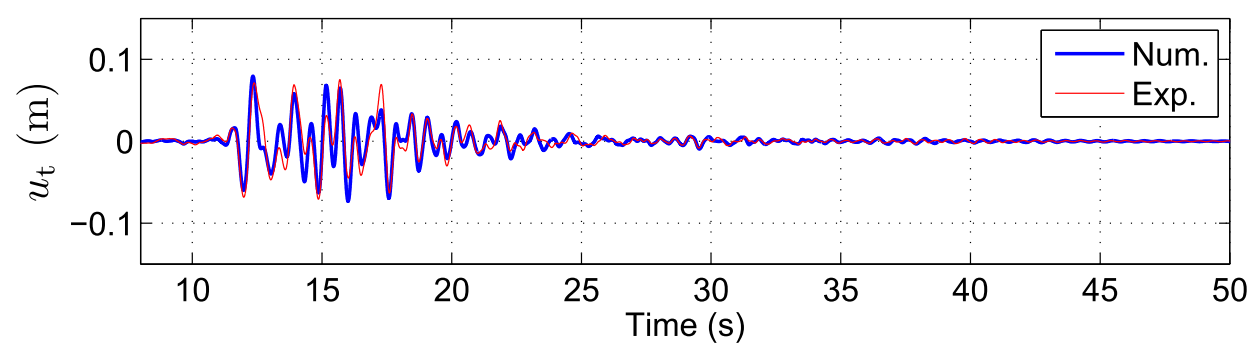

Fig. 22. Simulation of batter pile foundation with medium-tall superstructure ( $8.48 \mathrm{~m}$, Kobe input). Comparison of displacement history of top mass.

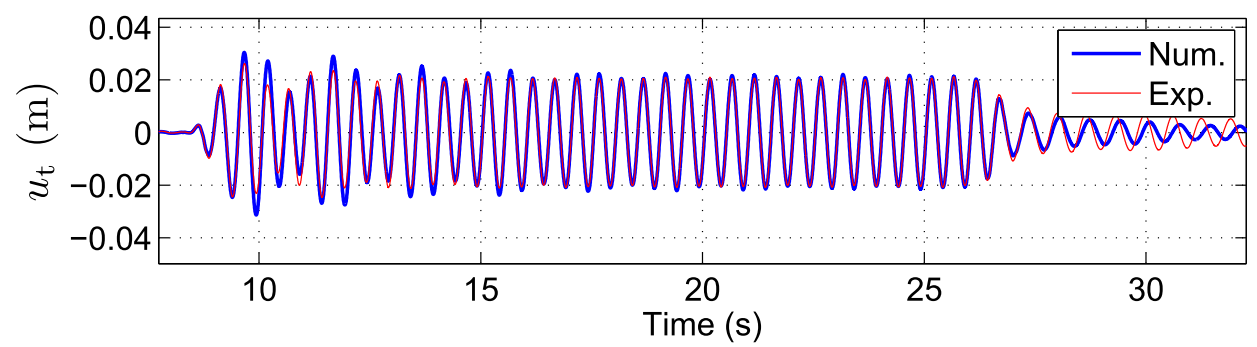

Fig. 23. Simulation of batter pile foundation with tall superstructure ( $14.16 \mathrm{~m}$, sine $2.0 \mathrm{~Hz}$ input). Comparison of displacement history of top mass.

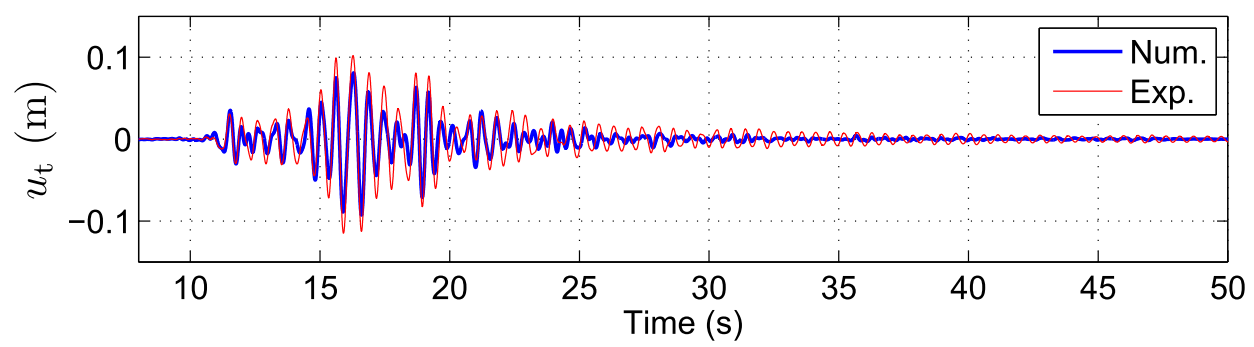

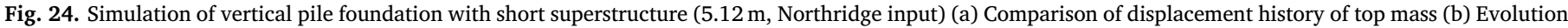
history of stiffness and damping ratio from their initial values.

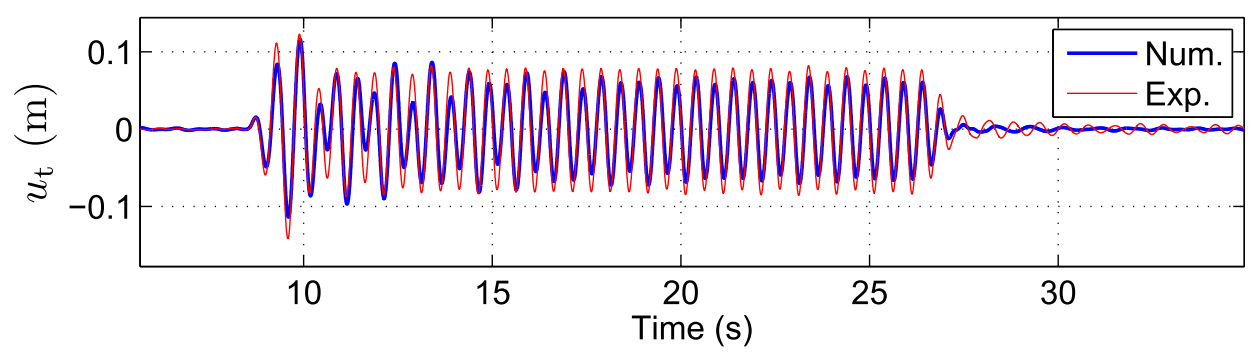

Fig. 25. Simulation of vertical pile foundation with tall superstructure ( $14.16 \mathrm{~m}$, sine $2.0 \mathrm{~Hz}$ input). Comparison of displacement history of top mass.

integration with constant average acceleration). For evaluating the effective displacement or rotation, the same factor i.e. 0.65 (in the original equivalent linear approach) is taken. In other words, the effective displacement or rotation of foundation is taken as $65 \%$ of their peak values. In the iterative procedure, the initial values of stiffness and damping ratios are taken the values listed in Tables 8 and 9. Maximum damping ratios were taken as the initial values to get a fast convergence for the iteration process. Then, the numerical model is validated and compared with the experimental results. For the dynamic inputs to the numerical model, the far-field accelerations measured at the ground surface were taken as the base excitation signals, see CH09 in Fig. 4.

Fig. 20 shows the comparison of model performance with experimental results. The simulated case is batter pile foundation with a short superstructure under $2.0 \mathrm{~Hz}$ excitation. A very good agreement is found between the numerical and experimental results. After several iterations, the stiffness and damping are stabilized as their final values, see Fig. 21(a). An example of iterative process of rotational stiffness is shown in Fig. 21(b). Other simulation cases are shown in Figs. 22-25. Equivalent linear analysis requires a number of iterations to obtain more accurate results. Although enough accuracy with differences of less than 5 to $10 \%$ are usually achieved in $3 \sim 5$ iterations [48], in this study, in order to further ensure the convergence of the iterative process, a large number of iterations was adopted. This large number is also recommended by similar code implementations (DeepSoil [49] recommends 15 as the number of iterations, for example).

From the results, it is proved that for batter (or vertical) foundation with short (or medium-tall and tall) superstructure, the numerical model has very good performance in reproducing the dynamic response of foundations under various dynamic loadings.

Numerical validation shows a good agreement with the experimental results. However, some limitation of the numerical simulations should be acknowledged. First of all, that equivalent linear approach that adopted in this study is not able to take account of the strong strain dependence to secant modulus and damping ratio. For large 
deformation under strong ground motions, using constant secant stiffness and damping ratio may not yield good results. Secondly, due to the limitation of accelerometers in the centrifuge tests, the residual displacement or rotation cannot be obtained which is also the case for equivalent linear approach. In this study, the good agreement of the comparisons only refers to dynamic displacements and rotations. However, despite these limitations, the proposed model may be utilized for preliminary evaluation of nonlinear SSI of pile foundations.

\section{Conclusions}

In this paper, the study of the performance of batter and vertical foundation in terms of translational/rotational stiffness and the damping properties is carried out. Empirical equations are also proposed to estimate the stiffness degradation and damping for foundations under dynamic loadings. It is shown that for the rocking behaviour, the presence of batter piles causes a small decrease of the the rotational stiffness and a great increase of the rotational damping; for the horizontal translation behaviour, batter pile foundation has much higher horizontal stiffness and translational damping compared to the vertical foundation. Finally, the proposed stiffness degradation and damping curves are implemented and integrated with equivalent linear approach. The numerical validation shows a good agreement with the experimental results. The feasibility of analysing the nonlinear response of pile foundations using simple stiffness degradation and damping curves is convinced. This work provides not only the insights to the stiffness degradation and energy dissipation properties of batter and vertical pile foundations, but also a simple and reliable numerical modelling approach for the design practice concerning the seismic response of deep foundations.

\section{Declaration of Competing Interest}

The authors declared that there is no conflict of interest.

\section{Acknowledgments}

Financial support from National Natural Science Foundation of China (NSFC, Grant No. 51708009) is highly acknowledged. The support of IFSTTAR (Institut français des sciences et technologies des transports, de l'aménagement et des réseaux) and of the Région Pays de la Loire is gratefully appreciated. The authors would like also to thank the valuable support and help of the technical staff of the IFSTTAR centrifuge team. The first author would acknowledge special thanks to Ms. Xuejiao Tang for her help.

\section{Appendix A. Rocking behavior of batter and vertical foundations}

\section{A.1. Rocking behavior of batter and vertical foundations under sine $3.5 \mathrm{~Hz}$ excitations}

See Figs. 26 and 27.

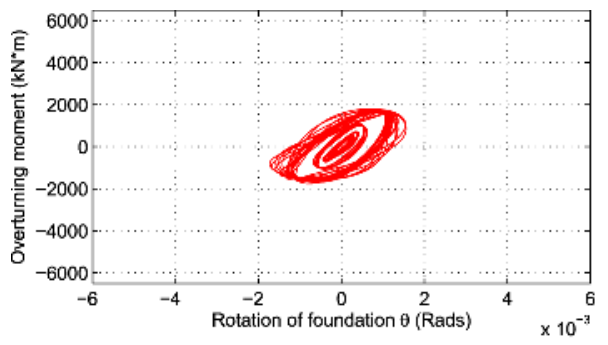

(a)

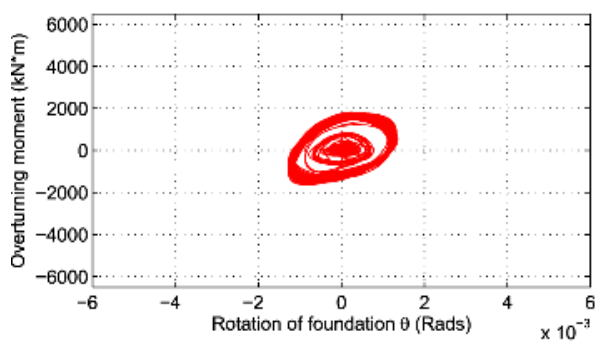

(c)

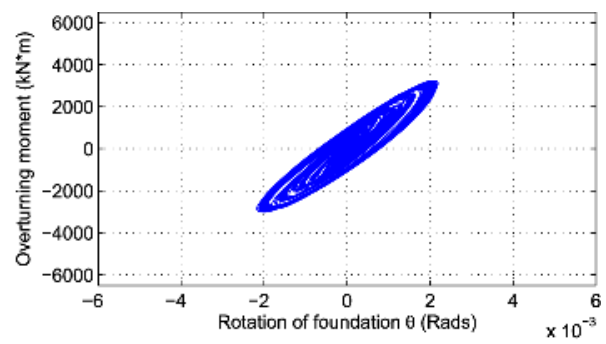

(b)

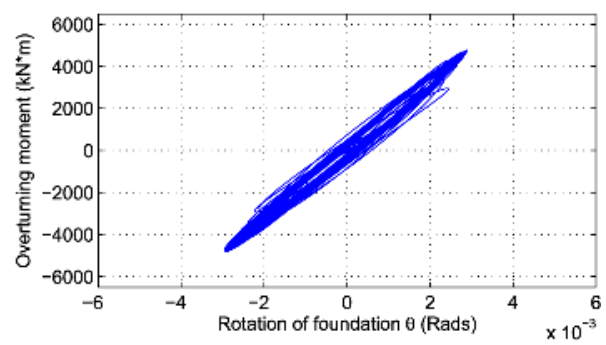

(d)

Fig. 26. Rotation-moment hysteretic loops, $3.5 \mathrm{~Hz}$ sine input (a) Batter pile foundation with short superstructure (5.12 m); (b) Vertical pile foundation with short superstructure $(5.12 \mathrm{~m})$; (c) Batter pile foundation with tall superstructure $(14.16 \mathrm{~m})$ and (d) Vertical pile foundation with tall superstructure $(14.16 \mathrm{~m})$. 


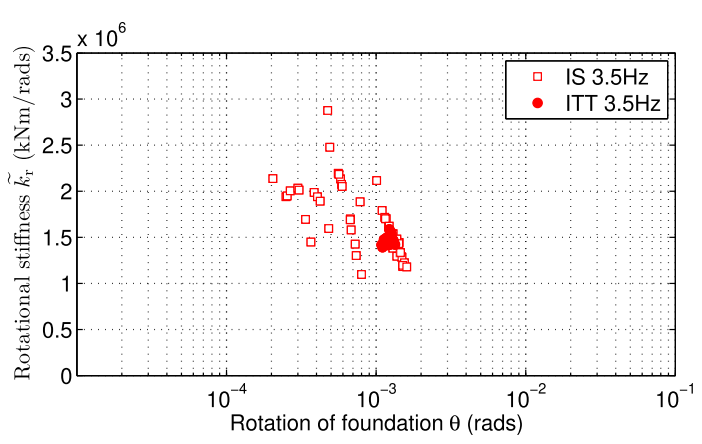

(a)

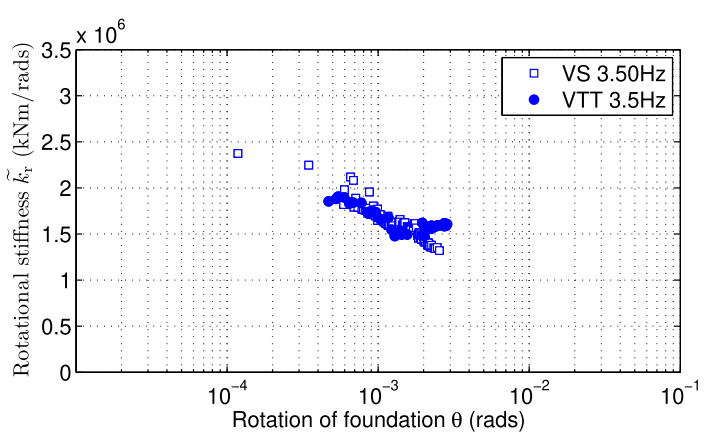

(c)

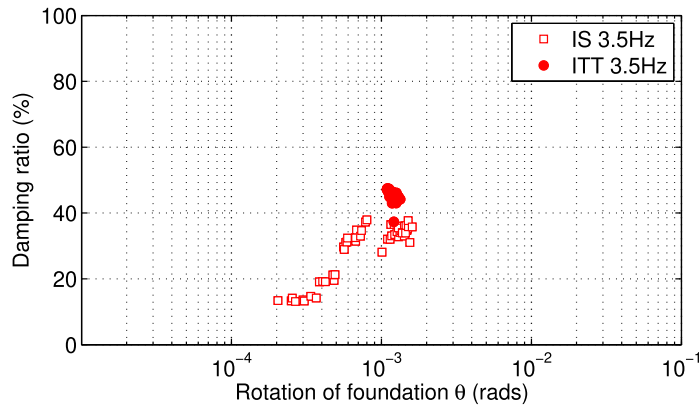

(b)

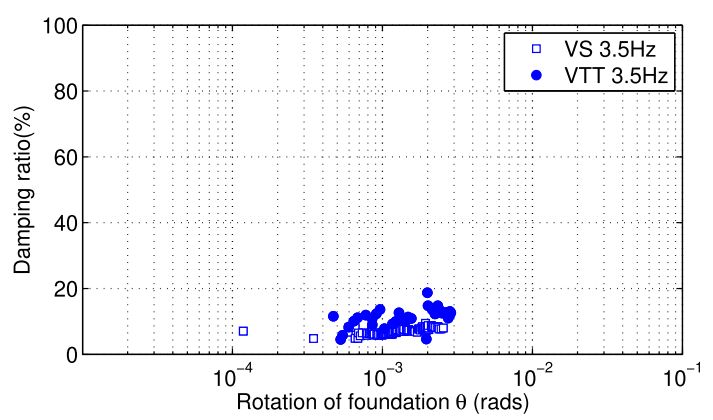

(d)

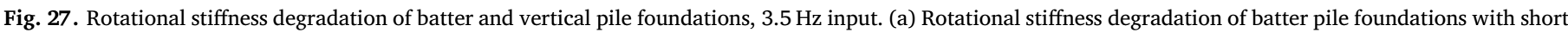

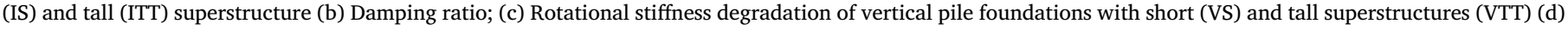
Damping ratio.

\section{A.2. Rocking behavior of batter and vertical foundations under real earthquake excitations}

See Figs. 28 and 29.

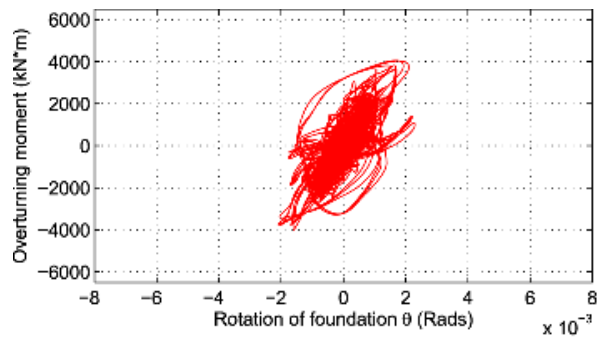

(a)

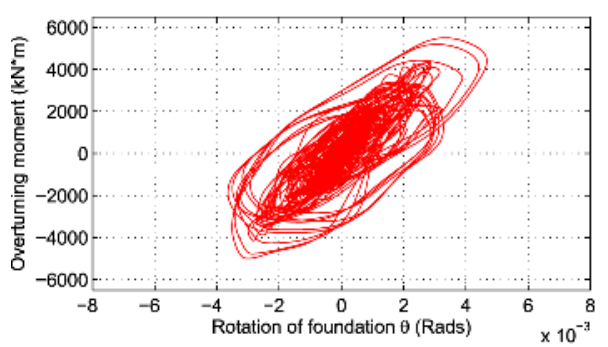

(c)

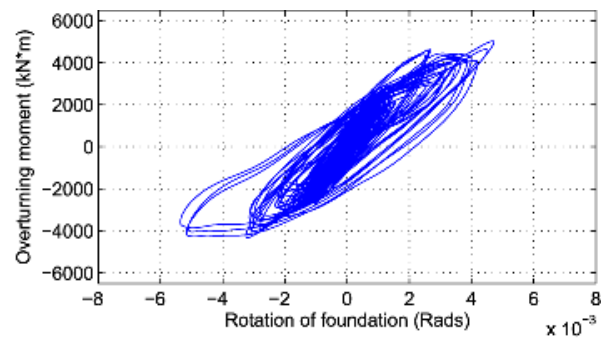

(b)

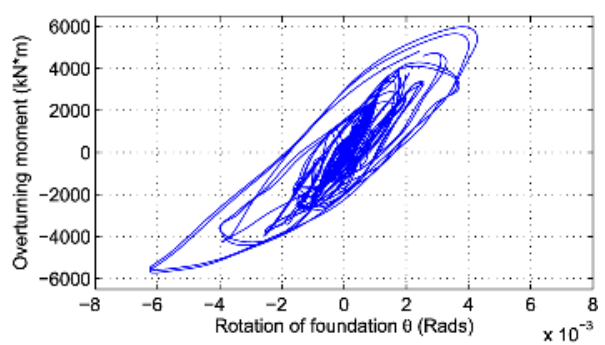

(d)

Fig. 28. Rotation-moment hysteretic loops, real earthquake input (a) Batter pile foundation with short superstructure (5.12 m); (b) Vertical pile foundation with short superstructure $(5.12 \mathrm{~m})$; (c) Batter pile group with medium-tall superstructure $(8.48 \mathrm{~m})$ and (d) Vertical pile foundation with medium-tall superstructure $(8.48 \mathrm{~m})$. 


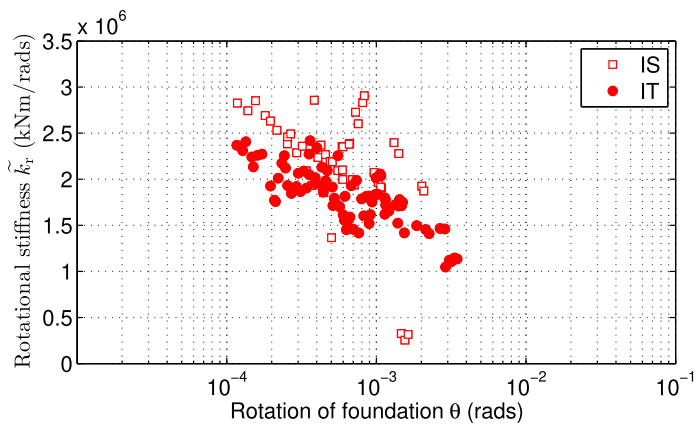

(a)

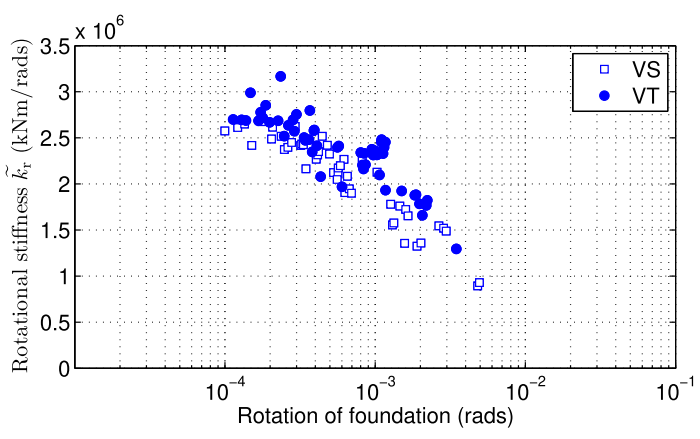

(c)

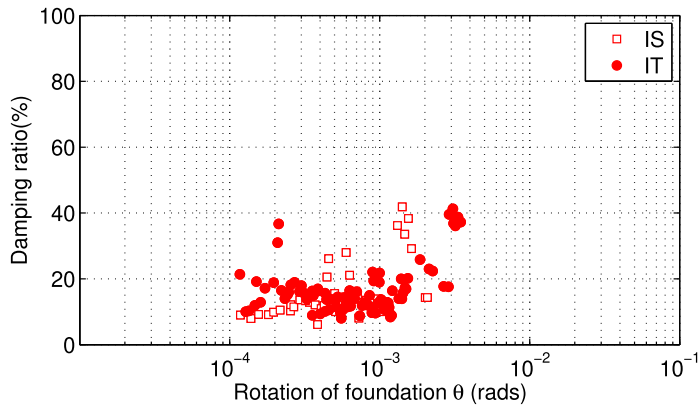

(b)

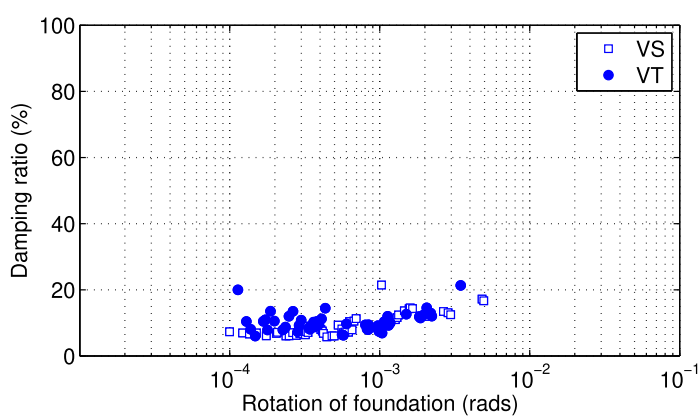

(d)

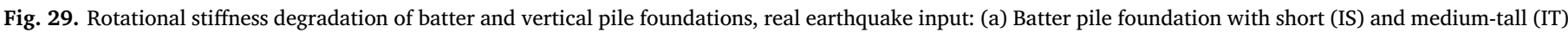
superstructure and (b) damping ratio; (c) Vertical pile foundation with short (VS) and medium-tall (VT) superstructures and (d) damping ratio.

\section{Appendix B. Lateral behavior of batter and vertical foundations}

\section{B.1. Lateral behavior of batter and vertical foundations under sine $2.0 \mathrm{~Hz}$ excitations}

See Figs. 30 and 31.

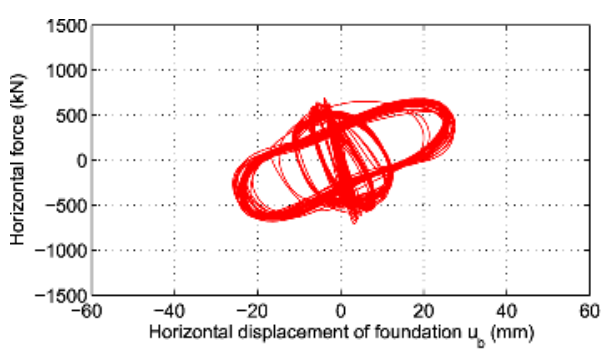

(a)

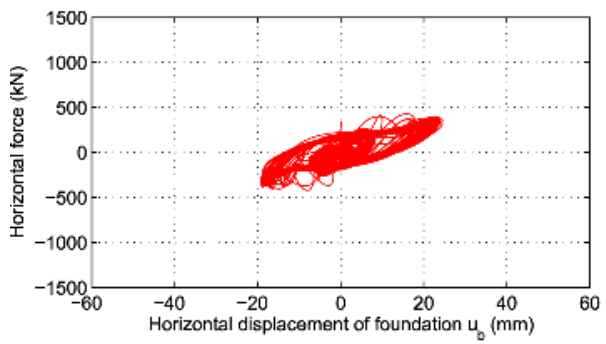

(c)

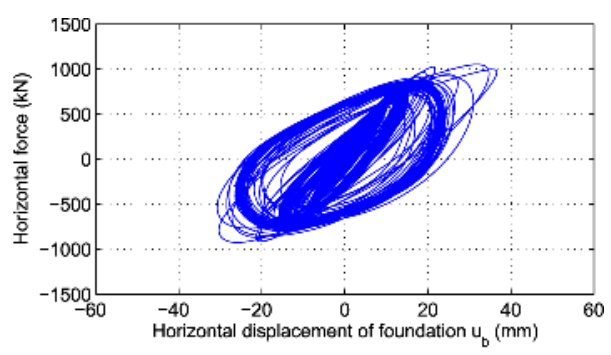

(b)

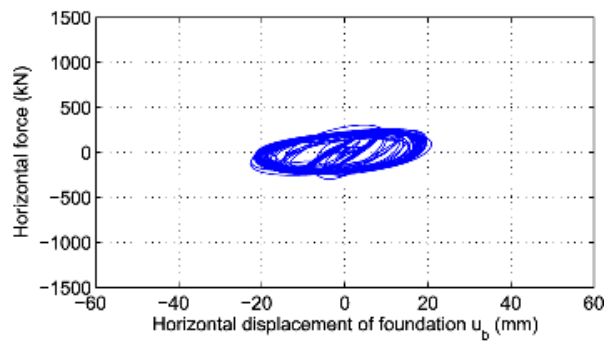

(d)

Fig. 30. Horizontal translation-force hysteretic loops, $2.0 \mathrm{~Hz}$ sine input: (a) Batter pile foundation with short superstructure (5.12 m); (b) Vertical pile foundation with short superstructure $(5.12 \mathrm{~m})$; (c) Batter pile foundation with tall superstructure $(14.16 \mathrm{~m})$ and (d) Vertical pile foundation with tall superstructure (14.16 m). 


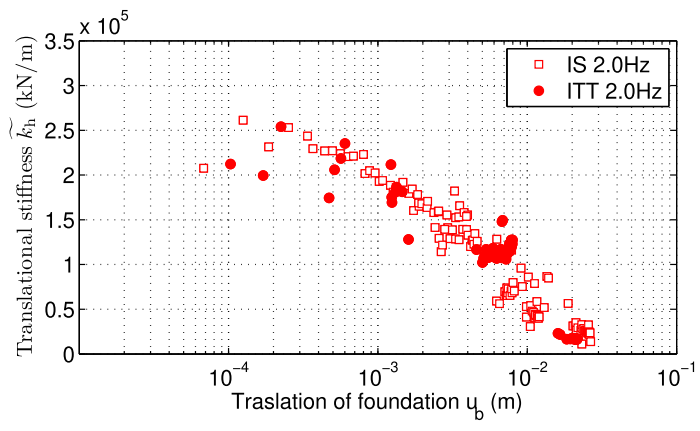

(a)

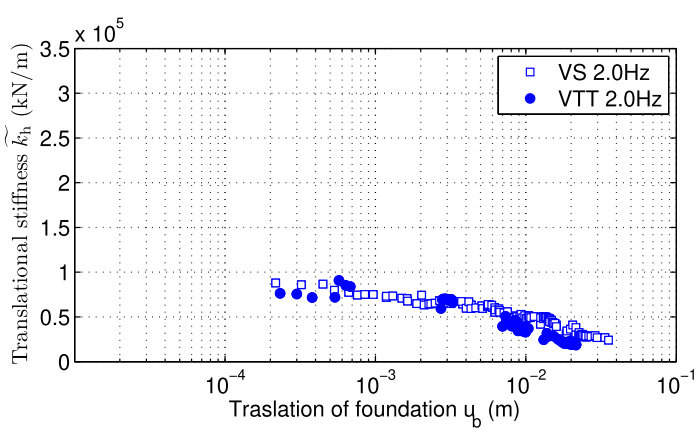

(c)

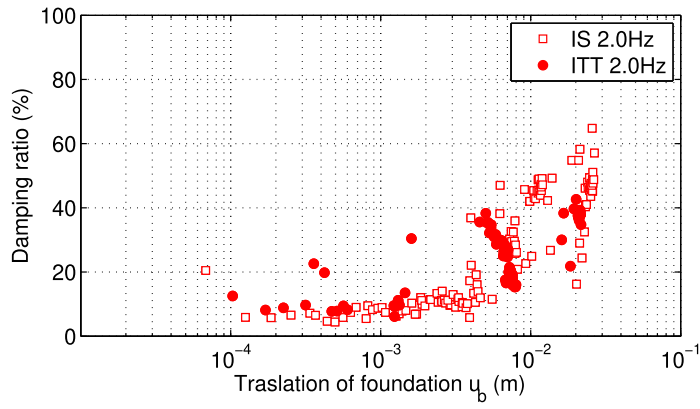

(b)

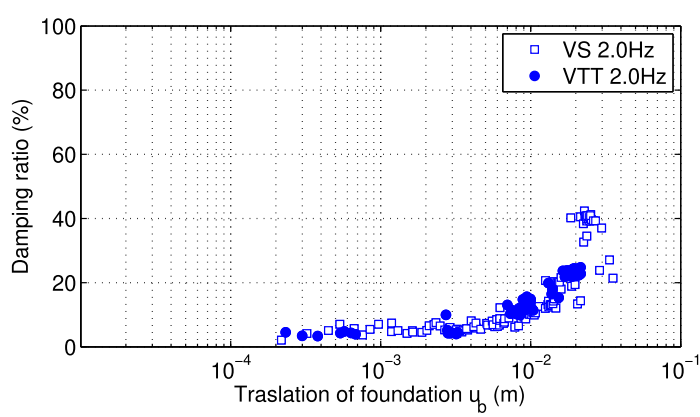

(d)

Fig. 31. Horizontal stiffness degradation of batter and vertical pile foundations, $2.0 \mathrm{~Hz}$ input: (a) Horizontal stiffness degradation of batter pile foundations with short (IS) and tall (ITT) superstructures (b) Damping ratio (c) Horizontal stiffness degradation of vertical pile foundations with short (VS) and tall superstructures (VTT) (d) Damping ratio.

\section{B.2. Lateral behavior of batter and vertical foundations under sine $3.5 \mathrm{~Hz}$ excitations}

See Figs. 32 and 33.

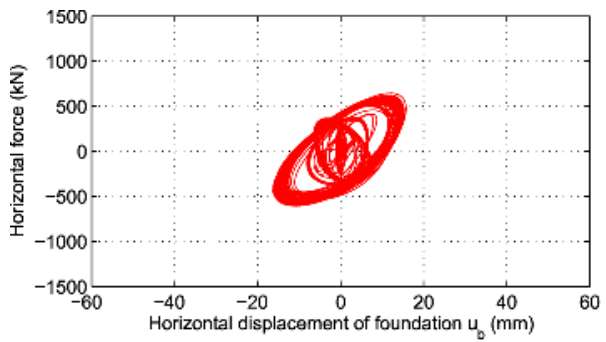

(a)

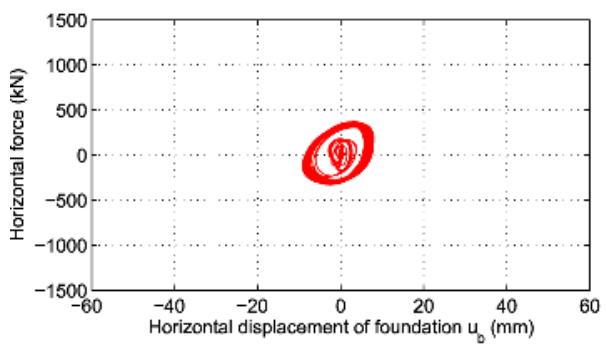

(c)

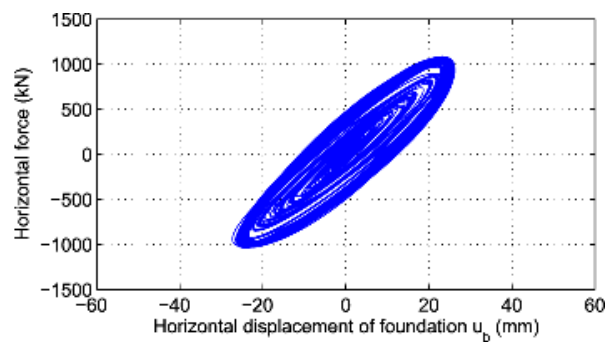

(b)

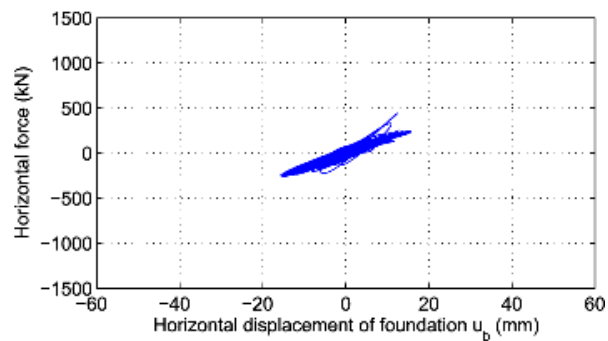

(d)

Fig. 32. Horizontal translation-force hysteretic loops, $3.5 \mathrm{~Hz}$ sine input (a) Batter pile group with short superstructure (5.12 m) (b) Vertical pile group with short superstructure $(5.12 \mathrm{~m})(\mathrm{c})$ Batter pile foundation with tall superstructure $(14.16 \mathrm{~m})$ and $(\mathrm{d})$ Vertical pile group with tall superstructure $(14.16 \mathrm{~m})$. 


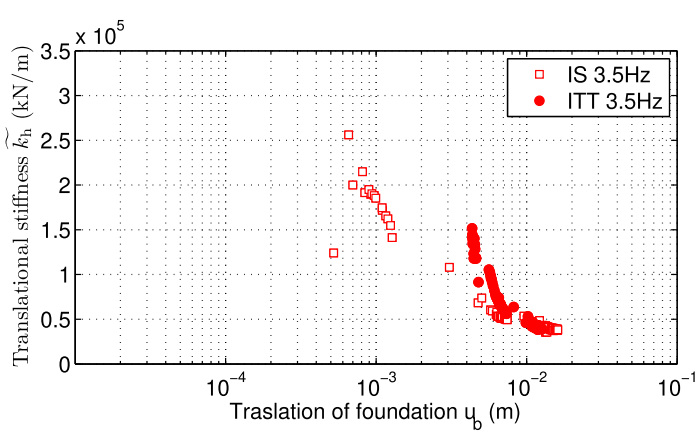

(a)

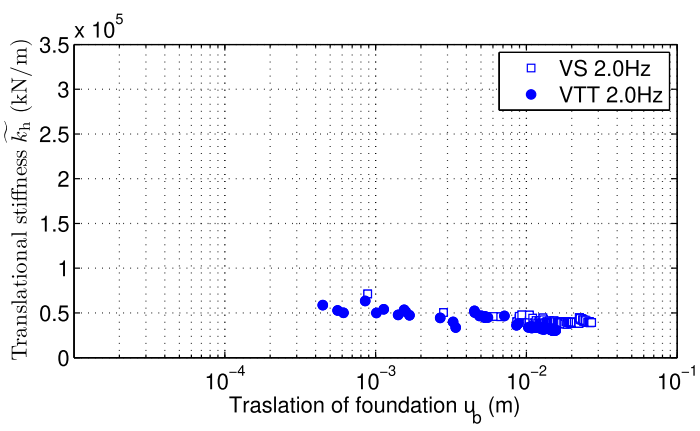

(c)

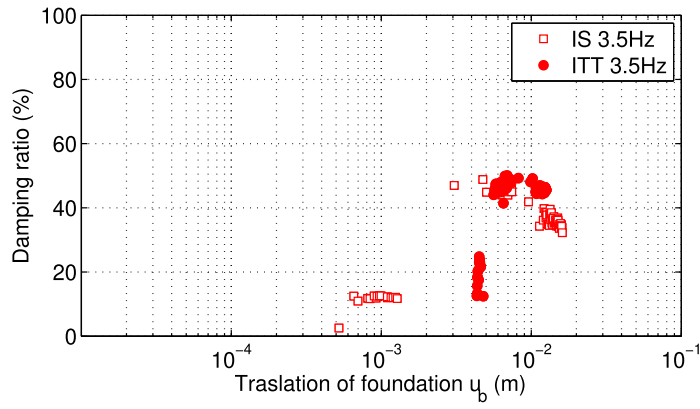

(b)

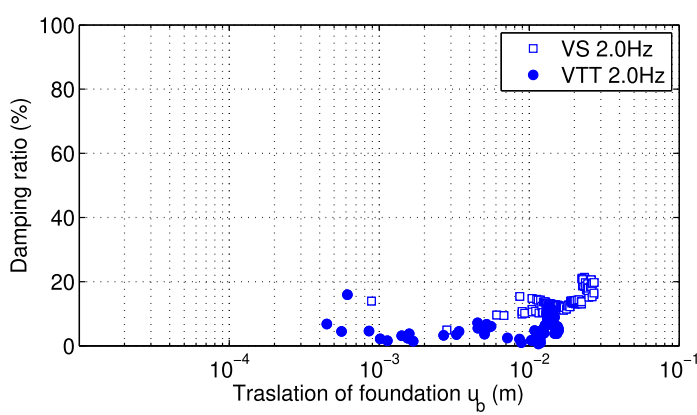

(d)

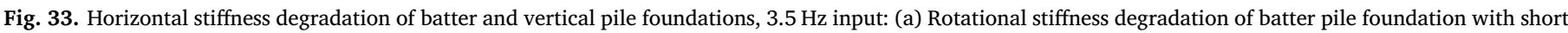

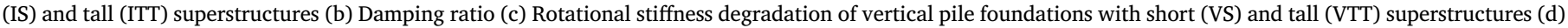
Damping ratio.

\section{B.2.1. Lateral behavior of batter and vertical foundations under real earthquake excitations}

See Figs. 34 and 35.

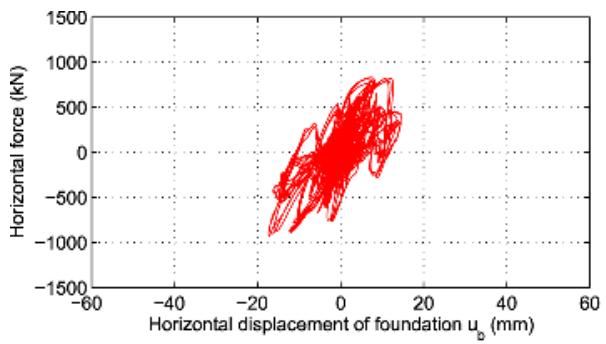

(a)

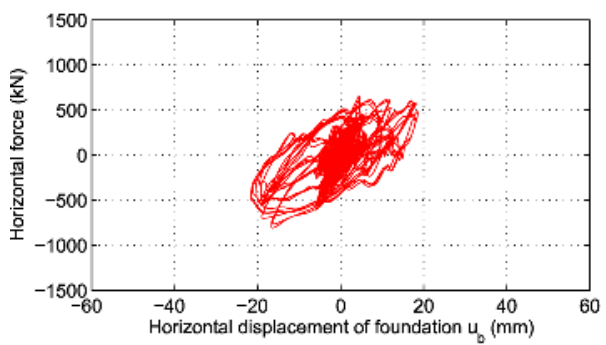

(c)

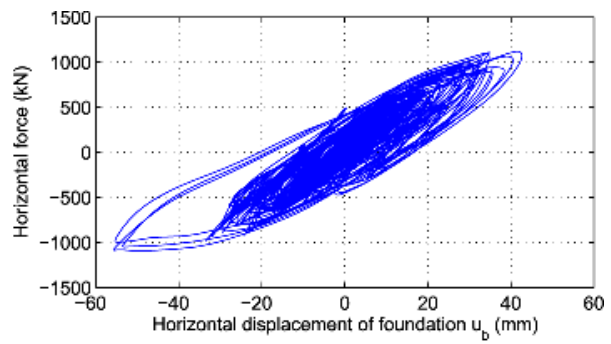

(b)

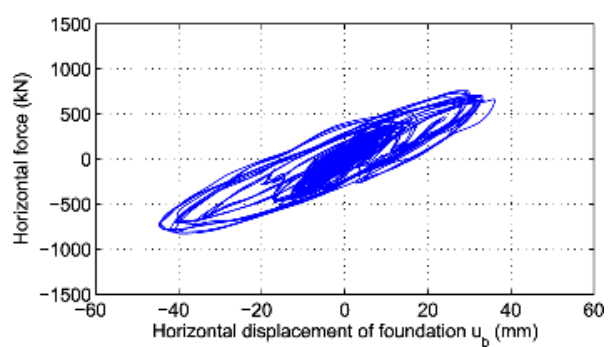

(d)

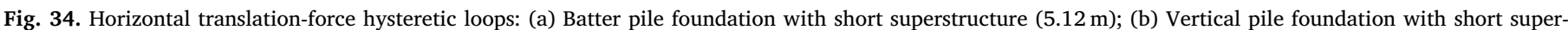

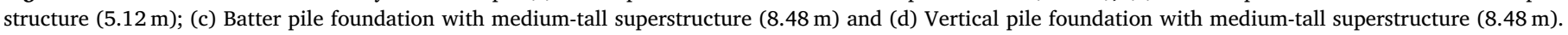




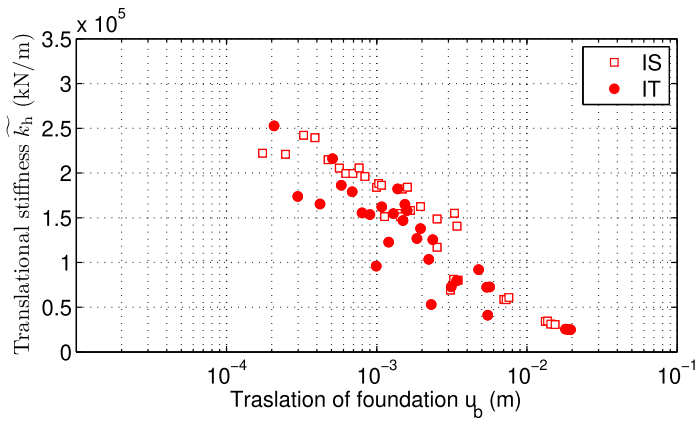

(a)

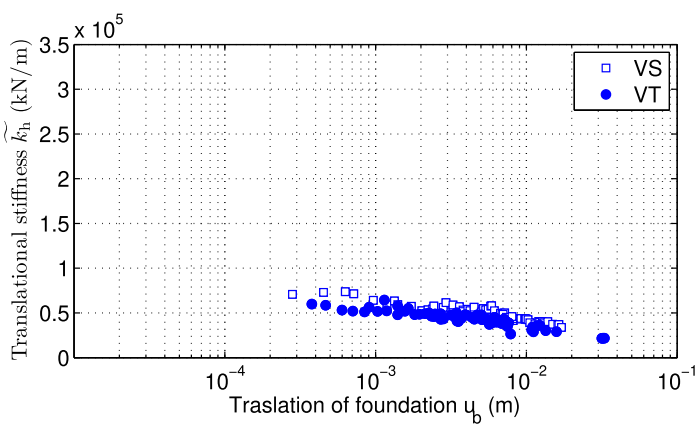

(c)

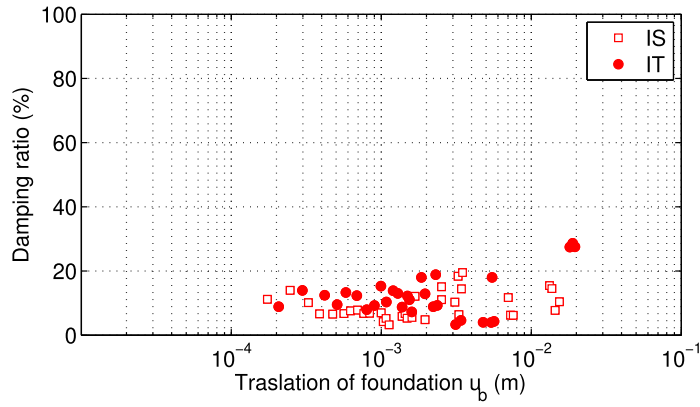

(b)

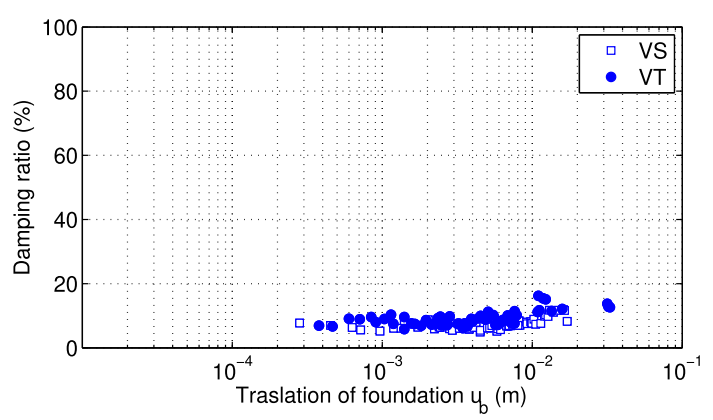

(d)

Fig. 35. Horizontal stiffness degradation of batter and vertical pile foundations, real earthquake input (a) Batter pile foundations with short (IS) and tall (ITT) superstructures and (b) damping ratio; (d) Vertical pile foundations with short (VS) and tall (VTT) superstructures and (d) damping ratio.

\section{References}

[1] Negro P, Paolucci R, Pedretti S, Faccioli E. Large scale soil-structure interaction experiments on sand under cyclic loading. 12th World conference on earthquake engineering. 2000. p. 1-8.

[2] Shirato M, Kouno T, Asai R, Nakatani S, Fukui J, Paolucci R. Large-scale experiments on nonlinear behavior of shallow foundations subjected to strong earthquakes. Soils Found 2008;48(5):673-92.

[3] Drosos V, Georgarakos T, Loli M, Anastasopoulos I, Zarzouras O, Gazetas G. Soilfoundation-structure interaction with mobilization of bearing capacity: experimental study on sand. J Geotech Geoenviron Eng 2012;138(11):1369-86. https:// doi.org/10.1061/(ASCE)GT.1943-5606.0000705.

[4] Deng L, Kutter BL, Kunnath SK. Seismic design of rocking shallow foundations: displacement-based methodology. J Bridge Eng 2014;19(11):04014043. https:// doi.org/10.1061/(ASCE)BE.1943-5592.0000616.

[5] Ahmadi E, Khoshnoudian F, Hosseini M. Importance of soil material damping in seismic responses of soil-MDOF structure systems. Soils Found 2015;55(1):35-44. https://doi.org/10.1016/j.sandf.2014.12.003.

[6] Pecker A, Paolucci R, Chatzigogos C, Correia AA, Figini R. The role of non-linear dynamic soil-foundation interaction on the seismic response of structures, Keynote Lecture. In: Second international conference on performance-based design in earthquake geotechnical engineering, Taormina, Italy; 2012. p. 1-19.

[7] Pecker A, Paolucci R, Chatzigogos C, Correia AA, Figini R. The role of non-linear dynamic soil-foundation interaction on the seismic response of structures. Bull Earthq Eng 2013;12:1157-76. https://doi.org/10.1007/s10518-013-9457-0.

[8] Cremer C, Pecker A, Davenne L. Cyclic macro-element for soil-structure interaction: material and geometrical non-linearities. Int J Numer Anal Meth Geomech 2001;25(13):1257-84. https://doi.org/10.1002/nag.175.

[9] Grange S, Kotronis P, Mazars J. A macro-element to simulate dynamic soil-structure interaction. Eng Struct 2009;31(12):3034-46. https://doi.org/10.1016/j.engstruct. 2009.08.007.

[10] Figini R, Paolucci R, Chatzigogos CT. A macro-element model for non-linear soilshallow foundation-structure interaction under seismic loads: theoretical development and experimental validation on large scale tests. Earthquake Eng Struct Dynam 2012;41:475-93. https://doi.org/10.1002/eqe.1140.

[11] Li Z, Kotronis P, Escoffier S, Tamagnini C. A hypoplastic macroelement for single vertical piles in sand subject to three-dimensional loading conditions. Acta Geotech 2016;11(2):373-90. https://doi.org/10.1007/s11440-015-0415-7.

[12] Lu Y, Marshall AM, Hajirasouliha I. A simplified Nonlinear Sway-Rocking model for evaluation of seismic response of structures on shallow foundations. Soil Dyn
Earthquake Eng 2016;81:14-26. https://doi.org/10.1016/j.soildyn.2015.11.002.

[13] Anastasopoulos I, Kontoroupi T. Simplified approximate method for analysis of rocking systems accounting for soil inelasticity and foundation uplifting. Soil Dyn Earthquake Eng 2014;56:28-43. https://doi.org/10.1016/j.soildyn.2013.10.001.

[14] Naggar MHE, Allotey N, Naggar MHE. Analytical moment - rotation curves for rigid foundations based on a Winkler model. Soil Dyn Earthquake Eng 2003;23:367-81. https://doi.org/10.1016/S0267-7261(03)00034-4.

[15] Tileylioglu S, Stewart J, Nigbor R. Dynamic stiffness and damping of a shallow foundation from forced vibration of a field test structure. J Geotech Geoenviron Eng 2011;1(4):344-53. https://doi.org/10.1061/(ASCE)GT.1943-5606.0000430.

[16] Anastasopoulos I, Kourkoulis R, Gelagoti F, Papadopoulos E. Rocking response of SDOF systems on shallow improved sand: an experimental study. Soil Dyn Earthquake Eng 2012;40:15-33. https://doi.org/10.1016/j.soildyn.2012.04.006.

[17] Kokkali P, Abdoun T, Anastasopoulos I. Centrifuge modeling of rocking foundations on improved soil. J Geotech Eeoenviron Eng 2015;141(10):1-15. https://doi.org/ 10.1061/(ASCE)GT.1943-5606.0001315.

[18] Gajan S, Kutter BL, Phalen JD, Hutchinson TC, Martin GR. Centrifuge modeling of load-deformation behavior of rocking shallow foundations. Soil Dyn Earthquake Eng 2005;25:773-83. https://doi.org/10.1016/j.soildyn.2004.11.019.

[19] Gazetas G, Anastasopoulos I, Adamidis O, Kontoroupi T. Nonlinear rocking stiffness of foundations. Soil Dyn Earthquake Eng 2013;47:83-91. https://doi.org/10.1016/ j.soildyn.2012.12.011

[20] Paolucci R, di Prisco C, Vecchiotti M, Shirato M, Yilmaz MT. Seismic behaviour of shallow foundations: large scale experiments vs. numerical modelling and implications for performance based design. In: Proceedings of the 1st US-Italy seismic bridge workshop, Eucentre, Pavia, Italy; 2007. p. 107-11.

[21] Paolucci R, Figini R, di Prisco C, Petrini L, Vecchiotti M. Accounting for non-linear dynamic soilstructure interaction in the displacement-based seismic design. In: Proceedings of the 5th international conference on earthquake geotechnical engineering., Santiago, Chile; 2011. p. 1-13.

[22] Paolucci R, Figini R, Petrini L. Introducing dynamic nonlinear soil-foundationstructure interaction effects in displacement-based seismic design. Earthquake Spectra 2013;29(2):475-96. https://doi.org/10.1193/1.4000135.

[23] Gazetas G. Analysis of machine foundation vibrations: state of the art. Int J Soil Dyn Earthquake Eng 1983;2(1):2-42. https://doi.org/10.1016/0261-7277(83)90025-6.

[24] Mergos PE, Kawashima K. Rocking isolation of a typical bridge pier on spread foundation. J Earthquake Eng 2005;9(2):395-414. https://doi.org/10.1142/ S1363246905002456.

[25] Chen Y-H, Liao W-H, Lee C-L, Wang Y-P. Seismic isolation of viaduct piers by means of a rocking mechanism. Earthquake Eng Struct Dynam 2006;35:713-36. https:// doi.org/10.1002/eqe.555. 
[26] Sullivan TJ. Soil-foundation-structure interaction considerations for performancebased design of RC wall structures on shallow foundations. In: Pender MJ, editor. Soil-foundation-structure interaction CRC Press; 2010. p. 193-200. https://doi.org/ 10.1201/b10568-29.

[27] Kourkoulis R, Anastasopoulos I, Gelagoti F, Kokkali P. Dimensional analysis of SDOF systems rocking on inelastic soil. J Earthquake Eng 2012;16(7):995-1022. https://doi.org/10.1080/13632469.2012.691615.

[28] Gelagoti F, Kourkoulis R, Anastasopoulos I, Gazetas G. Rocking isolation of low-rise frame structures founded on isolated footings. Earthquake Eng Struct Dynam 2012;41:1177-97. https://doi.org/10.1002/eqe.1182.

[29] Taeseri D, Laue J, Anastasopoulos I. Non-linear rocking stiffness of embedded foundations in sand. Géotechnique 2018:1-16. https://doi.org/10.1680/jgeot.17.P. 201.

[30] Adamidis O, Gazetas G, Anastasopoulos I, Argyrou C. Equivalent-linear stiffness and damping in rocking of circular and strip foundations. Bull Earthquake Eng 2014;12(3):1177-200. https://doi.org/10.1007/s10518-013-9554-0.

[31] Pitilakis D. Nonlinear soil-foundation-structure interaction aiming in performance based design. Proceedings of the international conference on performance-based design in earthquake geotechnical engineering, Tokyo. 2009. p. 1-7.

[32] Pitilakis D. Equivalent linear soil-foundation-structure interaction and performance based design. In: Earthquake geotechnical engineering satellite conference, XVIIth international conference on soil mechanics \& geotechnical engineering, Alexandria, Egypt; 2009. p. 1-9.

[33] Li Z, Escoffier S, Kotronis P. Centrifuge modeling of batter pile foundations under earthquake excitation. Soil Dyn Earthquake Eng 2016;88:176-90. https://doi.org/ 10.1016/j.soildyn.2016.05.013.

[34] Li Z, Escoffier S, Kotronis P. Centrifuge modeling of batter pile foundations under sinusoidal dynamic excitation. Bull Earthquake Eng 2016;14(3):673-97. https:// doi.org/10.1007/s10518-015-9859-2.

[35] Harris CM, Piersol AG. Harris' shock and vibration handbook. New York: McGrawHill; 2002.

[36] Chazelas JL, Escoffier S, Garnier J, Thorel L, Rault G. Original technologies for proven performances for the new LCPC earthquake simulator. Bull Earthquake Eng 2008;6(4):723-8. https://doi.org/10.1007/s10518-008-9096-z.

[37] Gazetas G, Dobry R. Simple radiation damping model for piles and footings. J Eng
Mech 1984;110(1):937-56. https://doi.org/10.1061/(ASCE)0733-9399(1984) 110:6(937).

[38] Çelebi M. Comparison of damping in buildings under low-amplitude and strong motions. J Wind Eng Ind Aerodyn 1996;59:309-23. https://doi.org/10.1016/0167 6105(96)00014-1.

[39] Celebi M. Response of olive view hospital to Northridge and Whittier earthquakes. J Struct Eng 1997;123(4):389-96.

[40] M. Çelebi, Radiation damping observed from seismic responses of buildings, in: 12th World Conference of Earthquake Engineering, 2000, p. 2634.

[41] Benaroya H, Nagurka ML, Han S. Mechanical vibration: analysis, uncertainties, and control. 4th ed. CRC Press; 2018.

[42] Kramer SL. Geotechnical earthquake engineering. New Jersey: Prentice Hall; 1996.

[43] Brennan A, Thusyanthan N, Madabhushi S. Evaluation of shear modulus and damping in dynamic centrifuge tests. J Geotech Geoenviron Eng 2005;131(12):1488-97. https://doi.org/10.1061/(ASCE)1090-0241(2005) 131:12(1488).

[44] Li Z, Escoffier S, Kotronis P. Using centrifuge tests data to identify the dynamic soil properties: application to Fontainebleau sand. Soil Dyn Earthquake Eng 2013;52:77-87. https://doi.org/10.1016/j.soildyn.2013.05.004.

[45] von Wolffersdorff P-A. A hypoplastic relation for granular materials with a predefined limit state surface. Mech Cohesive-Frict Mater 1996;1(4):251-71. https:// doi.org/10.1002/(SICI)1099-1484(199607)1:3 < 251::AID-CFM13 > 3.0.CO;2-3.

[46] Gudehus G, Amorosi A, Gens A, Herle I, Kolymbas D, Mašín D, et al. The soilmodels.info project. Int J Numer Anal Meth Geomech 2008;32(12):1571-2. https:// doi.org/10.1002/nag.675.

[47] Li Z, Kotronis P, Escoffier S, Tamagnini C. A hypoplastic macroelement formulation for single batter piles in sand. Int J Numer Anal Meth Geomech 2018;42(12):1346-65. https://doi.org/10.1002/nag. 2794.

[48] Schnabel P, Lysmer J, Seed H. SHAKE: a computer program for earthquake response analysis on horizontally layered sites, Tech. rep., Earthquake Engineering Research Center, Berkeley; 1972

[49] Hashash Y, Musgrove M, Harmon J, Groholski D, Phillips C, Park D. DEEPSOIL 6.1, User Manual, Tech. rep., Board of Trustees of University of Illinois at UrbanaChampaign., Urbana, IL; 2016. 